\title{
Chiral anomalies in six dimensions from harmonic superspace
}

\author{
Sergei M. Kuzenko, ${ }^{a}$ Joseph Novak $^{b}$ and Igor B. Samsonov ${ }^{c}$ \\ ${ }^{a}$ School of Physics and Astrophysics M013, The University of Western Australia, \\ 35 Stirling Highway, Crawley, W.A. 6009, Australia \\ ${ }^{b}$ Max-Planck-Institut für Gravitationsphysik, Albert-Einstein-Institut, \\ Am Mühlenberg 1, D-14476 Golm, Germany \\ ${ }^{c}$ Bogoliubov Laboratory of Theoretical Physics, JINR, \\ 141980 Dubna, Moscow region, Russia \\ E-mail: sergei.kuzenko@uwa.edu.au, joseph.novak@aei.mpg.de, \\ samsonov@theor. jinr.ru
}

ABSTRACT: We develop a superfield approach to compute chiral anomalies in general $\mathcal{N}=(1,0)$ supersymmetric gauge theories in six dimensions. Within the harmonicsuperspace formulation for these gauge theories, the anomalous contributions to the effective action only come from matter and ghost hypermultiplets. By studying the shortdistance behaviour of the propagator for the hypermultiplet coupled to a background vector multiplet, we compute the covariant and consistent chiral anomalies. We also provide a superform formulation for the non-abelian anomalous current multiplet in general $\mathcal{N}=(1,0)$ supersymmetric gauge theories.

Keywords: Anomalies in Field and String Theories, Extended Supersymmetry, Superspaces, Supersymmetric Gauge Theory

ARXIV EPRINT: 1708.08238 


\section{Contents}

1 Introduction 1

2 Superform formulation of the covariant chiral anomaly 3

2.1 The Yang-Mills multiplet 3

2.2 The linear multiplet 4

2.3 The non-abelian current multiplet 5

2.4 The anomalous non-abelian current multiplet 6

2.5 Another deformation of the non-abelian current multiplet $\quad 7$

3 Chiral anomaly in $\mathcal{N}=(1,0)$ harmonic superspace $\quad 8$

3.1 Effective action in non-anomalous gauge theories $\quad 8$

$\begin{array}{ll}3.2 & \text { Chiral anomaly and deformed conservation laws } \\ \end{array}$

$\begin{array}{lll}3.3 & \text { Consistent chiral anomaly in harmonic superspace } & 12\end{array}$

4 Chiral anomaly for the hypermultiplet effective action $\quad 14$

$\begin{array}{ll}4.1 \text { Hypermultiplet effective action } & 15\end{array}$

$\begin{array}{lll}4.2 & \text { Covariant chiral anomaly } & 18\end{array}$

$\begin{array}{lll}4.3 \text { Consistency terms } & 19\end{array}$

\begin{tabular}{ll}
4.4 Abelian limit 20 \\
\hline
\end{tabular}

5 Concluding comments $\quad 21$

$\begin{array}{ll}\text { A Vector multiplet in harmonic superspace } & 24\end{array}$

\section{Introduction}

The general structure of chiral and gravitational anomalies in gauge theories in diverse dimensions was fully understood as long ago as the mid-1980s [1-9] (more complete lists of references can be found, e.g., in [10,11]). In supersymmetric gauge theories, both chiral and gravitational anomalies (if present) should be embedded into supermultiplets. It is somewhat surprising that not much explicit information is available about the anomaly supermultiplets in six and higher spacetime dimensions, in contrast to four dimensions (4D). ${ }^{1}$ As is well known, the latter case is characterised by the absence of gravitational anomalies, while chiral gauge anomalies cannot occur in extended supersymmetry $(\mathcal{N}>1)$. We recall that the fermions in general $4 \mathrm{D} \mathcal{N}=2$ supersymmetric gauge theories transform in non-chiral representations of the gauge group.

\footnotetext{
${ }^{1}$ Chiral anomalies for general supersymmetric gauge theories in dimensions $D=6,8$ and 10 were computed in [12], and can be read off from the results in $[6,7,13-15]$, however no discussion of anomaly supermultiplets was given in these publications.
} 
The chiral anomalies in $4 \mathrm{D} \mathcal{N}=1$ supersymmetric gauge theories have been thoroughly studied in numerous works, see [16-27] and references therein. These studies include both the formal aspects of supersymmetric gauge anomalies as well as the powerful superfield techniques to compute such anomalies. In particular, it was realised that the (abelian) chiral anomaly can be viewed as a consistent deformation of the $\mathcal{N}=1$ linear multiplet $L=\bar{L}$. In the anomaly-free case, $L$ obeys the constraint $\bar{D}^{2} L=0$, which implies that the component field $\left.\left[D_{\alpha}, \bar{D}_{\dot{\alpha}}\right] L\right|_{\theta=0}$ is a conserved current [28]. If a chiral anomaly is present, the conservation equation is deformed to take the form

$$
\bar{D}_{\dot{\alpha}} \bar{D}^{\dot{\alpha}} L \propto W^{\alpha} W_{\alpha}
$$

where $W_{\alpha}$ is the chiral gauge-invariant field strength of a vector multiplet.

In the case of $6 \mathrm{D} \mathcal{N}=(1,0)$ supersymmetry, a conserved current belongs to the linear multiplet $L^{i j}$ which is a real $\mathrm{SU}(2)$ iso-triplet constrained by $D_{\alpha}^{(k} L^{i j)}=0$ [29]. In the presence of a chiral anomaly, this conservation equation turns into a deformed one. It was shown in our recent paper [30] (see also [31]) that the abelian chiral anomaly amounts to the following deformation

$$
A_{\alpha}^{i j k}:=D_{\alpha}^{(k} L^{i j)} \propto \mathrm{i} \varepsilon_{\alpha \beta \gamma \delta} W^{i \beta} W^{j \gamma} W^{k \delta},
$$

where $W^{i \alpha}$ is the gauge-invariant field strength of a vector multiplet. ${ }^{2}$ In [30] we also provided a nonlocal effective action which generates the anomaly (1.2). So far, however, even the abelian chiral anomaly (1.2) has never been computed directly in superspace in spite of the recent progress in applying the background covariant supergraphs to compute low-energy effective actions for $6 \mathrm{D} \mathcal{N}=(1,0)$ supersymmetric gauge theories [36-41]. The present paper aims to fill this gap by providing an explicit supergraph derivation of the non-abelian chiral anomaly using the $6 \mathrm{D} \mathcal{N}=(1,0)$ harmonic-superspace setting [42-44].

In $6 \mathrm{D} \mathcal{N}=(1,0)$ harmonic superspace, the non-abelian vector multiplet can be described either in terms of the analytic prepotential $V^{++}[42-44]$ (which is introduced in complete analogy with the $4 \mathrm{D} \mathcal{N}=2$ case [45]) or by means of an unconstrained harmonic superfield $M^{--}$defined by $V^{++}=\left(D^{+}\right)^{4} M^{--}[46]$. We will refer to $M^{--}$as the "generalised Mezincescu prepotential" due to the fact that $M^{--}$contains the $6 \mathrm{D}$ analogue $[32,33]$ of Mezincescu's prepotential $M^{i j}[47] .^{3}$ In order to compute the chiral anomalies using supergraphs, one may analyse the effective action $\Gamma=\Gamma\left[M^{--}\right]$in a general $6 \mathrm{D} \mathcal{N}=(1,0)$ supersymmetric gauge theory. Such a theory describes the pure supersymmetric YangMills (SYM) theory coupled to a hypermultiplet transforming in some representation of the gauge group. In this paper we argue that the non-abelian extension of (1.2) naturally originates as a covariant chiral anomaly. We also generalise Leutwyler's ideas [9] to the 6D $\mathcal{N}=(1,0)$ harmonic-superspace setting in order to construct a consistent chiral anomaly from the covariant one.

\footnotetext{
${ }^{2}$ The modern superfield formulation for $6 \mathrm{D} \mathcal{N}=(1,0)$ supersymmetric Yang-Mills theory was developed in $[32,33]$ as a reformulation of the earlier $\mathrm{SU}(2)$ non-covariant approach $[34,35]$.

${ }^{3}$ The prepotential $M^{--}$contains the conventional Mezincescu prepotential $M^{i j}$ as a leading Fourier coefficient in its harmonic expansion, $M^{--}(z, u)=M^{i j}(z) u_{i}^{-} u_{j}^{-}+\ldots$ As explained in [46], a gauge condition may be chosen in which $M^{--}(z, u)=M^{i j}(z) u_{i}^{-} u_{j}^{-}$.
} 
This paper is organised as follows. We first present a superform formulation of the non-abelian anomalous current multiplet in general $6 \mathrm{D} \mathcal{N}=(1,0)$ supersymmetric gauge theories in section 2. This construction allows for two possible forms of the non-abelian chiral anomaly, which reduce to the same expression in the abelian case. Explicit supergraph computation presented in subsequent sections shows that only one of these structures is realised as a part of the effective actions in $(1,0)$ gauge theories.

To compute chiral anomalies in general $6 \mathrm{D} \mathcal{N}=(1,0)$ supersymmetric gauge theories, it suffices to consider the hypermultiplet model in the presence of a background vector multiplet. Indeed, in pure SYM theory the anomalous contributions can only come from ghost superfields which are described by hypermultiplets according to [48-50]. Therefore, following the discussion of general aspects of covariant and consistent anomalies in superspace given in section 3 , in section 4 we examine the short-distance behaviour of the hypermultiplet propagator in the presence of a background vector multiplet and argue that the anomaly (1.2) naturally arises from those terms in the propagator which involve harmonic singularities. In the $4 \mathrm{D} \mathcal{N}=2$ and $5 \mathrm{D} \mathcal{N}=1$ cases, similar terms in the hypermultiplet propagator give no contributions to the effective action [51,52], in agreement with the fact that all $4 \mathrm{D} \mathcal{N}=2$ supersymmetric gauge theories have no chiral anomaly (which is also obviously true for the $5 \mathrm{D} \mathcal{N}=1$ theories). This uncovers the role of harmonic singularities in the context of chiral anomalies.

In the main body of the paper, we mostly study the form of the chiral anomaly in the formulation of the $(1,0)$ gauge theory based on the generalised Mezincescu prepotential $M^{--}$. However, the concluding section 5 discusses the issues of describing the chiral anomaly in the formulation of gauge theory with the analytic prepotential $V^{++}$. In particular, we propose a consistent expression for this anomaly in the abelian case.

In this paper, we follow the 6D superspace notation and conventions given in [53] and employed in our recent paper [30]. In appendix $\mathrm{A}$ we review the basic aspects of the $6 \mathrm{D}$ supersymmetric gauge theories in $\mathcal{N}=(1,0)$ harmonic superspace.

\section{Superform formulation of the covariant chiral anomaly}

In our recent paper [30], we presented a superform formulation of the anomalous current multiplet for the abelian chiral anomaly. In this section, we generalise those results to the case of the non-abelian chiral anomaly. In particular, a superform formulation of the covariant anomalous current multiplet will be developed. For the reader's convenience, we start this section with a short review of the superform descriptions of the 6D Yang-Mills and linear multiplets.

\subsection{The Yang-Mills multiplet}

In this subsection we review the superspace formulation for the $6 \mathrm{D} \mathcal{N}=(1,0)$ Yang-Mills supermultiplet. To describe a non-abelian vector multiplet, the covariant derivative of Minkowski superspace $D_{A}=\left(\partial_{a}, D_{\alpha}^{i}\right)$ has to be replaced with a gauge-covariant one,

$$
\mathcal{D}_{A}:=D_{A}+\mathrm{i} V_{A}
$$


Here the gauge connection one-form $V=\mathrm{d} z^{A} V_{A}$ takes its values in the Lie algebra of the Yang-Mills gauge group. The covariant derivative algebra is

$$
\left[\mathcal{D}_{A}, \mathcal{D}_{B}\right\}=T_{A B}{ }^{C} \mathcal{D}_{C}+\mathrm{i} F_{A B},
$$

where the only non-vanishing torsion is

$$
T_{\alpha \beta}^{i j c}=-2 \mathrm{i} \varepsilon^{i j}\left(\gamma^{c}\right)_{\alpha \beta}
$$

and $F_{A B}$ are the components of the gauge covariant field strength two-form $F=\mathrm{d} z^{B} \wedge$ $\mathrm{d} z^{A} F_{A B}$. The covariant derivatives and field strength may be written in a coordinate-free way as follows

$$
\mathcal{D}=\mathrm{d}+\mathrm{i} V, \quad F=\mathrm{d} V-\mathrm{i} V \wedge V,
$$

where we have introduced $\mathcal{D}:=\mathrm{d} z^{A} \mathcal{D}_{A}$. The field strength $F$ satisfies the Bianchi identity

$$
\mathcal{D} F=\mathrm{d} F+\mathrm{i} V \wedge F-\mathrm{i} F \wedge V=0 \quad \Longleftrightarrow \quad \mathcal{D}_{[A} F_{B C\}}-T_{[A B}^{D} F_{|D| C\}}=0 .
$$

The Yang-Mills gauge transformation acts on the gauge covariant derivatives $\mathcal{D}_{A}$ and a matter superfield $U$ (transforming in some representation of the gauge group) as

$$
\mathcal{D}_{A} \rightarrow \mathrm{e}^{\mathrm{i} \tau} \mathcal{D}_{A} \mathrm{e}^{-\mathrm{i} \tau}, \quad U \rightarrow U^{\prime}=\mathrm{e}^{\mathrm{i} \tau} U, \quad \tau^{\dagger}=\tau,
$$

where the Hermitian gauge parameter $\tau(z)$ takes its values in the Lie algebra of the gauge group. This implies that the gauge connection and field strength transform as follows

$$
V \rightarrow \mathrm{e}^{\mathrm{i} \tau} V \mathrm{e}^{-\mathrm{i} \tau}-\mathrm{ie}^{\mathrm{i} \tau} \mathrm{de}^{-\mathrm{i} \tau}, \quad F \rightarrow \mathrm{e}^{\mathrm{i} \tau} F \mathrm{e}^{-\mathrm{i} \tau} .
$$

Some components of the field strength two-form have to be constrained in order to describe an irreducible multiplet. Upon constraining the lowest mass dimension component of the field strength two-form as

$$
F_{\alpha \beta}^{i j}=0, \quad F_{a \beta}^{j}=\left(\gamma_{a}\right)_{\beta \gamma} W^{j \gamma},
$$

the remaining component is determined from the Bianchi identity (2.5) to be

$$
F_{a b}=-\frac{\mathrm{i}}{8}\left(\gamma_{a b}\right)_{\beta}{ }^{\alpha} \mathcal{D}_{\alpha}^{k} W_{k}^{\beta}
$$

and the superfield $W^{i \alpha}$ is required to obey the differential constraints

$$
\mathcal{D}_{\gamma}^{k} W_{k}^{\gamma}=0, \quad \mathcal{D}_{\alpha}^{(i} W^{j) \beta}=\frac{1}{4} \delta_{\alpha}^{\beta} \mathcal{D}_{\gamma}^{(i} W^{j) \gamma} .
$$

\subsection{The linear multiplet}

It is instructive to first describe the conserved current multiplet in the abelian case which invariably is described by a linear multiplet (or $\mathcal{O}(2)$ multiplet). The linear multiplet can be described using a four-form gauge potential $B=\frac{1}{4 !} \mathrm{d} z^{D} \wedge \mathrm{d} z^{C} \wedge \mathrm{d} z^{B} \wedge \mathrm{d} z^{A} B_{A B C D}$ possessing the gauge transformation

$$
\delta B=\mathrm{d} \rho
$$


where the gauge parameter $\rho$ is an arbitrary three-form. The corresponding field strength is

$$
H=\mathrm{d} B=\frac{1}{5 !} \mathrm{d} z^{E} \wedge \mathrm{d} z^{D} \wedge \mathrm{d} z^{C} \wedge \mathrm{d} z^{B} \wedge \mathrm{d} z^{A} H_{A B C D E}
$$

where

$$
H_{A B C D E}=5 D_{[A} B_{B C D E\}}-10 T_{[A B}^{F} B_{|F| C D E\}} .
$$

The field strength must satisfy the Bianchi identity

$$
\mathrm{d} H=0 \quad \Longleftrightarrow \quad D_{[A} H_{B C D E F\}}-\frac{5}{2} T_{[A B}{ }^{G} H_{|G| C D E F\}}=0 .
$$

In order to describe the linear multiplet we need to impose some covariant constraints on the field strength $H$. We choose the constraint

$$
H_{a b c \alpha \beta}^{i j}=-2 \mathrm{i}\left(\gamma_{a b c}\right)_{\alpha \beta} L^{i j}, \quad L^{i j}=L^{j i},
$$

and require all lower dimension components to vanish. We can now solve for the remaining components of $H$ in terms of $L^{i j}$. The solution is

$$
\begin{aligned}
H_{a b c d \alpha}{ }^{i} & =-\frac{1}{6} \varepsilon_{a b c d e f}\left(\gamma^{e f}\right)_{\alpha}^{\beta} D_{\beta j} L^{i j}, \\
H_{a b c d e} & =-\frac{\mathrm{i}}{24} \varepsilon_{a b c d e f}\left(\tilde{\gamma}^{f}\right)^{\alpha \beta} D_{\alpha}^{k} D_{\beta}^{l} L_{k l},
\end{aligned}
$$

where $L^{i j}$ is required to satisfy the constraint for the linear multiplet

$$
D_{\alpha}^{(i} L^{j k)}=0
$$

\subsection{The non-abelian current multiplet}

A non-abelian current multiplet described by a superfield $L^{i j}=L^{(i j)}$ must take values in the Lie algebra of the gauge group and satisfy the constraint

$$
\mathcal{D}_{\alpha}^{(i} L^{j k)}=0
$$

To find a superform formulation we need to introduce a five-form $\mathcal{H}$ built out of $L^{i j}$ such that its superform equation is satisfied as a result of the conservation equation above. To do this we write down the superform equation

$$
\mathcal{D H}-\Sigma=0,
$$

where $\Sigma$ is some covariant six-form taking values in the Lie algebra of the gauge group. Consistency of the above equation requires

$$
\mathcal{D} \Sigma-[F, \mathcal{H}]=0 \Longrightarrow \mathcal{D}_{\left[A_{1}\right.} \Sigma_{\left.A_{2} \cdots A_{7}\right\}}-3 T_{\left[A_{1} A_{2}\right.}{ }^{B} \Sigma_{\left.|B| A_{3} \cdots A_{7}\right\}}=3\left[F_{\left[A_{1} A_{2}\right.}, \mathcal{H}_{\left.A_{3} \cdots A_{7}\right\}}\right]
$$

The equation (2.21) does not have a bosonic analogue since a seven-form in six dimensions vanishes, but it becomes an important requirement in the supersymmetric case where it 
demands that there exists a covariant solution to equation $(2.21) .{ }^{4}$ One can check that there exists a covariant solution to (2.21) and its non-vanishing components are:

$$
\begin{aligned}
\Sigma_{a b c d e \alpha}^{i} & =-\varepsilon_{a b c d e f}\left(\gamma^{f}\right)_{\alpha \beta}\left[W_{j}^{\beta}, L^{i j}\right], \\
\Sigma_{a b c d e f} & =\frac{\mathrm{i}}{16} \varepsilon_{a b c d e f}\left(\left[\mathcal{D}_{\alpha}^{i} W^{j \alpha}, L_{i j}\right]-\frac{8}{3}\left\{W_{i}^{\alpha}, \mathcal{D}_{\alpha j} L^{i j}\right\}\right) .
\end{aligned}
$$

The superform $\mathcal{H}$ possesses the following non-vanishing components:

$$
\begin{aligned}
\mathcal{H}_{a b c \alpha \beta}{ }^{i j} & =-2 \mathrm{i}\left(\gamma_{a b c}\right)_{\alpha \beta} L^{i j}, \\
\mathcal{H}_{a b c d \alpha} & =-\frac{1}{6} \varepsilon_{a b c d e f}\left(\gamma^{e f}\right)_{\alpha}{ }^{\beta} \mathcal{D}_{\beta j} L^{i j}, \\
\mathcal{H}_{a b c d e} & =-\frac{\mathrm{i}}{24} \varepsilon_{a b c d e f}\left(\tilde{\gamma}^{f}\right)^{\alpha \beta} \mathcal{D}_{\alpha}^{k} \mathcal{D}_{\beta}^{l} L_{k l} .
\end{aligned}
$$

The superform equations (2.20) and (2.21) are satisfied as a consequence of the constraint (2.19).

\subsection{The anomalous non-abelian current multiplet}

To describe the covariant anomaly one only needs to modify the superform equation (2.20) as follows

$$
\mathcal{D H}-\Sigma=\kappa \operatorname{str}\left(T^{\mathcal{A}} F \wedge F \wedge F\right) T_{\mathcal{A}}=\kappa d^{\mathcal{A B C D}} F_{\mathcal{A}} \wedge F_{\mathcal{B}} \wedge F_{\mathcal{C}} T_{\mathcal{D}}
$$

where $\kappa$ is some constant and 'str' is the symmetrised trace. The two-form field strength $F$ takes its values in the Lie algebra of the gauge group with generators $T^{\mathcal{A}}$

$$
F=F_{\mathcal{A}} T^{\mathcal{A}}
$$

and $d^{\mathcal{A B C D}}$ is the gauge-invariant tensor

$$
d^{\mathcal{A B C D}}=\operatorname{tr}\left(T^{(\mathcal{A}} T^{\mathcal{B}} T^{\mathcal{C}} T^{\mathcal{D})}\right) .
$$

The solution to the superform equation (2.24) is just a deformation of the solution in the previous subsection. It turns out that only two components of $\mathcal{H}$ must be modified and they are given by

$$
\begin{aligned}
\mathcal{H}_{a b c d_{\alpha}}^{i}= & -\frac{1}{6} \varepsilon_{a b c d e f}\left(\gamma^{e f}\right)_{\alpha}{ }^{\beta} \mathcal{D}_{\beta j} L^{i j} \\
& +\kappa \mathrm{i} \varepsilon_{a b c d e f}\left(\gamma^{e}\right)_{\alpha \beta}\left(\gamma^{f}\right)_{\gamma \delta} \operatorname{str}\left(T^{\mathcal{A}} W_{j}^{\beta} W^{(j \gamma} W^{i) \delta}\right) T_{\mathcal{A}}, \\
\mathcal{H}_{a b c d e}= & \varepsilon_{a b c d e f} \tilde{\mathcal{H}}^{f}
\end{aligned}
$$

where

$$
\begin{aligned}
\tilde{\mathcal{H}}^{a}= & -\frac{\mathrm{i}}{24}\left(\tilde{\gamma}^{a}\right)^{\alpha \beta} \mathcal{D}_{\alpha}^{k} \mathcal{D}_{\beta}^{l} L_{k l}+\frac{\kappa}{8} \operatorname{str}\left(T^{\mathcal{A}}\left(\mathcal{D}_{\gamma k} W_{l}^{\gamma}\right)\left(W^{k} \gamma^{a} W^{l}\right)\right) T_{\mathcal{A}} \\
& +\frac{3 \kappa \mathrm{i}}{8} \operatorname{str}\left(T^{\mathcal{A}} F_{b c}\left(W^{k} \gamma^{a b c} W_{k}\right)\right) T_{\mathcal{A}} .
\end{aligned}
$$

\footnotetext{
${ }^{4}$ This requirement is known in the literature as Weil triviality [54].
} 
The superform equation (2.24) requires that $L^{i j}$ satisfy the differential equation

$$
\begin{aligned}
\mathcal{D}_{\alpha}^{(i} L^{j k)} & =\kappa \mathrm{i} \varepsilon_{\alpha \beta \gamma \delta} \operatorname{str}\left(W^{(i \beta} W^{j \gamma} W^{k) \delta} T^{\mathcal{A}}\right) T_{\mathcal{A}} \\
& =\kappa \mathrm{i} \varepsilon_{\alpha \beta \gamma \delta} d^{\mathcal{A B C D}} W_{\mathcal{A}}^{(i \beta} W_{\mathcal{B}}^{j \gamma} W_{\mathcal{C}}^{k) \delta} T_{\mathcal{D}} .
\end{aligned}
$$

The value of the constant $\kappa$ will be fixed by the explicit calculation in the next section. In particular, for the model of a hypermultiplet interacting with a background Yang-Mills multiplet this constant is $\kappa=-\frac{1}{96 \pi^{3}}$.

The highest dimension component of (2.24) implies the following

$$
\mathcal{D}_{a} \tilde{\mathcal{H}}^{a}-\frac{\mathrm{i}}{16}\left(\left[\mathcal{D}_{\alpha}^{i} W^{j \alpha}, L_{i j}\right]-\frac{8}{3}\left\{W_{i}^{\alpha}, \mathcal{D}_{\alpha j} L^{i j}\right\}\right)=\frac{\kappa}{8} \varepsilon^{a b c d e f} \operatorname{str}\left(T^{\mathcal{A}} F_{a b} F_{c d} F_{e f}\right) T_{\mathcal{A}} .
$$

Thus the component projection of $\tilde{\mathcal{H}}^{a}$ should be understood as the current whose conservation condition is now deformed.

\subsection{Another deformation of the non-abelian current multiplet}

It is worth mentioning that besides the deformation just considered, there exists another deformation of the current multiplet. To describe it, one modifies the superform equation (2.20) as follows

$$
\mathcal{D H}-\Sigma=\mu \operatorname{tr}(F \wedge F) \wedge F,
$$

where $\mu$ is some constant. The superform equation (2.31) is solved by

$$
\begin{aligned}
\mathcal{H}_{a b c \alpha \beta}^{i j} & =-2 \mathrm{i}\left(\gamma_{a b c}\right)_{\alpha \beta} L^{i j}, \\
\mathcal{H}_{a b c d \alpha} & =-\frac{1}{6} \varepsilon_{a b c d e f}\left(\gamma^{e f}\right)_{\alpha}{ }^{\beta} \mathcal{D}_{\beta j} L^{i j}+\mu \mathrm{i} \varepsilon_{a b c d e f}\left(\gamma^{e}\right)_{\alpha \beta}\left(\gamma^{f}\right)_{\gamma \delta} \operatorname{tr}\left(W^{(i \gamma} W^{j) \delta}\right) W_{j}^{\beta}, \\
\mathcal{H}_{a b c d e} & =\varepsilon_{a b c d e f} \tilde{\mathcal{H}}^{f}
\end{aligned}
$$

where

$$
\tilde{\mathcal{H}}^{a}=-\frac{\mathrm{i}}{24}\left(\tilde{\gamma}^{a}\right)^{\alpha \beta} \mathcal{D}_{\alpha}^{k} \mathcal{D}_{\beta}^{l} L_{k l}+\frac{\mu}{8} \operatorname{tr}\left(W^{k} \gamma^{a} W^{l}\right) \mathcal{D}_{\gamma k} W_{l}^{\gamma}+\frac{3 \mu \mathrm{i}}{8} \operatorname{tr}\left(W^{k} \gamma^{a b c} W_{k}\right) F_{b c}
$$

and all lower dimension components of $\mathcal{H}$ vanish. The superfield $L^{i j}$ is now required to satisfy the differential equation

$$
\mathcal{D}_{\alpha}^{(i} L^{j k)}=\mu \mathrm{i} \varepsilon_{\alpha \beta \gamma \delta} \operatorname{tr}\left(W^{(i \beta} W^{j \gamma}\right) W^{k) \delta} .
$$

The highest dimension component of eq. (2.31) implies

$$
\mathcal{D}_{a} \tilde{\mathcal{H}}^{a}-\frac{\mathrm{i}}{16}\left(\left[\mathcal{D}_{\alpha}^{i} W^{j \alpha}, L_{i j}\right]-\frac{8}{3}\left\{W_{i}^{\alpha}, \mathcal{D}_{\alpha j} L^{i j}\right\}\right)=\frac{\mu}{8} \varepsilon^{a b c d e f} \operatorname{tr}\left(F_{a b} F_{c d}\right) F_{e f} .
$$

The component projection of $\tilde{\mathcal{H}}^{a}$ corresponds to the current with a deformed conservation equation as described above. It should be mentioned that although this deformation exists it is not physically realised except in the abelian case where it coincides with the previous deformation. 


\section{Chiral anomaly in $\mathcal{N}=(1,0)$ harmonic superspace}

In this section we discuss the general aspects of covariant and consistent anomalies in $6 \mathrm{D} \mathcal{N}=(1,0)$ supersymmetric gauge theories in the harmonic superspace. The relation between the standard superspace employed above and the harmonic one is outlined in appendix A.

\subsection{Effective action in non-anomalous gauge theories}

Let us consider an anomaly-free gauge theory in $\mathcal{N}=(1,0)$ harmonic superspace. The effective action $\Gamma$ of such a theory may always be chosen to be a gauge-invariant functional, $\Gamma=\Gamma\left[V^{++}\right]$, of the analytic gauge prepotential $V^{++}=V_{\mathcal{A}}^{++} T^{\mathcal{A}}$ taking its values in the Lie algebra of the gauge group. The generators of the gauge group, $T^{\mathcal{A}}$, will be normalised so that $\operatorname{tr}_{(\mathrm{F})}\left(T^{\mathcal{A}} T^{\mathcal{B}}\right)=\delta^{\mathcal{A B}}$ in the defining representation. If the prepotential is perturbed, $V^{++} \rightarrow V^{++}+\delta V^{++}$, the variation of the effective action can be represented in the form

$$
\delta \Gamma=\int \mathrm{d} \zeta^{(-4)} \delta V_{\mathcal{A}}^{++} L^{++\mathcal{A}}=\operatorname{tr}_{(\mathrm{F})} \int \mathrm{d} \zeta^{(-4)} \delta V^{++} L^{++},
$$

for some effective current $L^{++}=L_{\mathcal{A}}^{++} T^{\mathcal{A}}=L^{++}\left(V^{++}\right)$obeying the analyticity constraint

$$
D_{\alpha}^{+} L^{++}=0 \text {. }
$$

The effective action is invariant under infinitesimal gauge transformations

$$
\delta_{\lambda} V^{++}=-\mathcal{D}^{++} \lambda,
$$

where the gauge parameter $\lambda=\lambda_{\mathcal{A}} T^{\mathcal{A}}$ is analytic, $D_{\alpha}^{+} \lambda=0$.

The invariance condition $\delta_{\lambda} \Gamma=0$ implies that the effective current obeys the harmonic shortness constraint

$$
\mathcal{D}^{++} L^{++}=0 \text {. }
$$

The general solution to this constraint reads

$$
L^{++}=\mathrm{e}^{\mathrm{i} b} L_{\tau}^{++} \mathrm{e}^{-\mathrm{i} b}, \quad L_{\tau}^{++}(z, u)=L^{i j}(z) u_{i}^{+} u_{j}^{+},
$$

where $L^{i j}(z)$ obeys, as a consequence of (3.1b), the conservation equation

$$
\mathcal{D}_{\alpha}^{(i} L^{j k)}=0 \text {. }
$$

Here $b=b(z, u)$ is the bridge superfield, see appendix A for the technical details.

The analyticity constraint $D_{\alpha}^{+} V^{++}=0$ can always be solved as

$$
V^{++}=\left(D^{+}\right)^{4} M^{--},
$$

where $M^{--}$is an unconstrained superfield (subject to a certain reality condition) on the full harmonic superspace. It is defined modulo gauge transformations

$$
\delta_{\xi} M^{--}=D_{\alpha}^{+} \xi^{(-3) \alpha},
$$


which do not change $V^{++}$for any unconstrained gauge parameter $\xi^{(-3) \alpha}$. We emphasise that (3.2) and (3.7) are two different gauge symmetries. The gauge transformation (3.7) is absent when one works solely with $V^{++}$.

The vector multiplet can be described either in terms of $V^{++}$or in terms of $M^{--}$. We will refer to these descriptions as $V$-formulation and $M$-formulation, respectively. The gauge freedom in the $V$-formulation is given by (3.2). Let us now discuss, in some more detail, the gauge freedom in the $M$-formulation.

When dealing with $M^{--}$, it is natural to express the analytic gauge parameter $\lambda$ in (3.2) via an unconstrained one to be denoted $\rho^{(-4)}$,

$$
\lambda=\left(D^{+}\right)^{4} \rho^{(-4)} .
$$

Then the $\lambda$-transformation (3.2) results in the following variation of $M^{--}$

$$
\delta_{\lambda} M^{--}=-\mathcal{D}^{++} \rho^{(-4)},
$$

modulo a $\xi$-transformation (3.7). The complete gauge freedom in the $M$-formulation is

$$
\delta M^{--}=-\mathcal{D}^{++} \rho^{(-4)}+D_{\alpha}^{+} \xi^{(-3) \alpha} .
$$

The prepotential $M^{--}$has the following harmonic expansion:

$$
M^{--}(z, u)=M^{i j}(z) u_{i}^{-} u_{j}^{-}+\sum_{\kappa=1}^{\infty} M^{\left(i_{1} \ldots i_{k} j_{1} \ldots j_{k+2}\right)}(z) u_{i_{1}}^{+} \ldots u_{i_{k}}^{+} u_{j_{1}}^{-} \ldots u_{j_{k+2}}^{-} .
$$

A similar series can be introduced for the gauge parameter $\rho^{(-4)}$

$$
\rho^{(-4)}(z, u)=\sum_{\kappa=0}^{\infty} \rho^{\left(i_{1} \ldots i_{k} j_{1} \ldots j_{k+4}\right)}(z) u_{i_{1}}^{+} \ldots u_{i_{k}}^{+} u_{j_{1}}^{-} \ldots u_{j_{k+4}}^{-} .
$$

Then the transformation law (3.9) tells us that all the superfields $M^{\left(i_{1} \ldots i_{k} j_{1} \ldots j_{k+2}\right)}, k \geq 1$, in the Fourier series (3.11) can be gauged away algebraically. In the resulting gauge

$$
M^{--}(z, u)=M^{i j}(z) u_{i}^{-} u_{j}^{-}
$$

the local symmetry (3.9) is completely fixed. However, we still have the freedom to perform $\xi$-transformations (3.7) generated by a single harmonic-independent parameter $\xi^{i j k \alpha}(z)$, which originates as $\xi^{(-3) \alpha}(z, u)=\frac{4}{3} \xi^{i j k \alpha}(z) u_{i}^{-} u_{j}^{-} u_{k}^{-}$. The gauge superfield $M^{i j}(z)$ may be recognised as Mezincescu's prepotential [32, 33, 47]. We will refer to the unconstrained superfield $M^{--}(z, u)$ defined by (3.6) as the generalised Mezincescu prepotential.

The above discussion shows that all the superfields $M^{\left(i_{1} \ldots i_{k} j_{1} \ldots j_{k+2}\right)}, k \geq 1$, in the Fourier series (3.11) may be interpreted as compensators, for all of them can be gauged away algebraically by applying local transformations (3.9). ${ }^{5}$ Thus in the $M$-formulation, the local $\xi$-invariance (3.7) plays the role of genuine gauge transformations while the $\rho$ gauge freedom (3.9) becomes purely compensating. The significance of this observation is

\footnotetext{
${ }^{5}$ One of the most familiar examples of compensators is the scalar field of the Stueckelberg formulation, which is used to introduce a local gauge invariance in the theory with a massive vector field.
} 
that, in general, the compensating gauge symmetries are known to be non-anomalous [55]. This means that in the $M$-formulation the presence of chiral anomalies is equivalent to the fact that the local $\xi$-invariance (3.7) becomes anomalous.

For our subsequent discussion, it is instructive to look at the gauge transformations of $M^{--}$in the $\tau$-frame. By construction, the relation (3.6) is defined in the $\lambda$-frame, where the gauge-covariant spinor derivative $\mathcal{D}_{\alpha}^{+}$has no gauge connection, $\mathcal{D}_{\alpha}^{+}=D_{\alpha}^{+}$. If the analytic prepotential is subject to a perturbation, $V^{++} \rightarrow V^{++}+\delta V^{++}$, then the generalised Mezincescu prepotential also changes, $M^{--} \rightarrow M^{--}+\delta M^{--}$, such that

$$
\delta V^{++}=\left(D^{+}\right)^{4} \delta M^{--} .
$$

This relation in the $\tau$-frame becomes

$$
\delta V_{\tau}^{++}=\left(\mathcal{D}^{+}\right)^{4} \delta M_{\tau}^{--},
$$

where

$$
\delta V_{\tau}^{++}=\mathrm{e}^{-\mathrm{i} b} \delta V^{++} \mathrm{e}^{\mathrm{i} b}, \quad \delta M_{\tau}^{--}=\mathrm{e}^{-\mathrm{i} b} \delta M^{--} \mathrm{e}^{\mathrm{i} b} .
$$

For $\delta M_{\tau}^{--}$the gauge transformations (3.7) and (3.9) read, respectively,

$$
\begin{aligned}
\delta_{\xi} M_{\tau}^{--} & =\mathcal{D}_{\alpha}^{+} \xi_{\tau}^{(-3) \alpha}, \\
\delta_{\lambda} M_{\tau}^{--} & =-D^{++} \rho_{\tau}^{(-4)},
\end{aligned}
$$

where

$$
\xi_{\tau}^{(-3) \alpha}=\mathrm{e}^{-\mathrm{i} b} \xi^{(-3) \alpha} \mathrm{e}^{\mathrm{i} b}, \quad \rho_{\tau}^{(-4)}=\mathrm{e}^{-\mathrm{i} b} \rho^{(-4)} \mathrm{e}^{\mathrm{i} b} .
$$

Unlike the original gauge transformations (3.7) and (3.9) in the $\lambda$-frame, the spinor derivative in (3.17a) is gauge covariant, while the harmonic derivative in (3.17b) has no gauge connection.

In the $\tau$-frame, the superfields $\delta M_{\tau}^{--}, \xi_{\tau}^{(-3) \alpha}$ and $\rho_{\tau}^{(-4)}$ have the following harmonic expansions:

$$
\begin{aligned}
\delta M_{\tau}^{--}(z, u) & =\delta M^{i j}(z) u_{i}^{-} u_{j}^{-}+\sum_{\kappa=1}^{\infty} \delta M^{\left(i_{1} \ldots i_{k} j_{1} \ldots j_{k+2}\right)}(z) u_{i_{1}}^{+} \ldots u_{i_{k}}^{+} u_{j_{1}}^{-} \ldots u_{j_{k+2}}^{-} \\
\xi_{\tau}^{(-3) \alpha}(z, u) & =\frac{4}{3} \xi^{i j k \alpha}(z) u_{i}^{-} u_{j}^{-} u_{k}^{-}+\ldots \\
\rho_{\tau}^{(-4)}(z, u) & =\sum_{\kappa=0}^{\infty} \rho_{\tau}^{\left(i_{1} \ldots i_{k} j_{1} \ldots j_{k+4}\right)}(z) u_{i_{1}}^{+} \ldots u_{i_{k}}^{+} u_{j_{1}}^{-} \ldots u_{j_{k+4}}^{-}
\end{aligned}
$$

It is clear that the gauge freedom (3.17b) may be used to impose a gauge condition

$$
\delta M_{\tau}^{--}(z, u)=\delta M^{i j}(z) u_{i}^{-} u_{j}^{-} .
$$

The residual gauge transformations, which preserve the gauge, are generated by

$$
\xi_{\tau}^{(-3) \alpha}(z, u)=\frac{4}{3} \xi^{i j k \alpha}(z) u_{i}^{-} u_{j}^{-} u_{k}^{-}, \quad \rho^{(-4)}(z, u)=\frac{1}{3} \mathcal{D}_{\alpha}^{i} \xi^{j k l \alpha} u_{i}^{-} u_{j}^{-} u_{k}^{-} u_{l}^{-} .
$$


In accordance with (3.17a), the Mezincescu prepotential transforms as

$$
\delta_{\xi} M_{i j}=\mathcal{D}_{\alpha}^{k} \xi_{i j k}^{\alpha} .
$$

One can use the operator $\left(D^{+}\right)^{4}$ in $(3.14)$ to restore the full superspace measure in (3.1a) and to represent the variation of the effective action in two equivalent forms

$$
\delta \Gamma=\operatorname{tr}_{(\mathrm{F})} \int \mathrm{d}^{6 \mid 8} z \mathrm{~d} u \delta M^{--} L^{++}=\operatorname{tr}_{(\mathrm{F})} \int \mathrm{d}^{6 \mid 8} z \mathrm{~d} u \delta M_{\tau}^{--} L_{\tau}^{++},
$$

where the effective current $L_{\tau}^{++}$is given by eq. (3.4). In the $\tau$-frame, the harmonic integral can be easily computed to result with

$$
\delta \Gamma=\frac{1}{3} \int \mathrm{d}^{6 \mid 8} z \delta M_{\mathcal{A}}^{i j} L_{i j}^{\mathcal{A}} .
$$

The invariance of the effective action under the $\xi$-transformations $(3.24), \delta_{\xi} \Gamma=0$, is equivalent to the analyticity constraint on the effective current $L^{i j}$,

$$
\mathcal{D}_{\alpha}^{(i} L^{j k)}=0
$$

The $\lambda$ (or $\rho$ ) gauge freedom disappears once the gauge condition (3.22) has been chosen.

\subsection{Chiral anomaly and deformed conservation laws}

As was pointed out in the previous subsection, in non-anomalous gauge theories the effective current $L^{++}$obeys the constraints of Grassmann analyticity (3.1b) and harmonic shortness (3.3). Either of these constraints may, in principle, be violated in theories which suffer from chiral anomalies depending on which of the gauge transformations, (3.2) or (3.7), becomes broken. We recall that eq. (3.2) describes the gauge freedom in the $V$-formulation while in the $M$-formulation the gauge freedom is larger and is given by (3.10). In the latter case the $\rho$-transformation is compensating and, therefore, non-anomalous [55]. It is the $\xi$-transformations which are anomalous in the $M$-formulation.

In those supersymmetric gauge theories which suffer from chiral anomalies at the quantum level, the $V$-formulation and the $M$-formualtion become non-equivalent as they are described by two different effective currents which we denote by $L_{\mathrm{an}}^{++}$and $L_{\mathrm{Mez}}^{++}$, respectively.

In the $V$-formulation, the effective current $L_{\text {an }}^{++}$remains analytic while the harmonic shortness constraint (3.3) may be broken

$$
\mathcal{D}_{\alpha}^{+} L_{\mathrm{an}}^{++}=0, \quad \mathcal{D}^{++} L_{\mathrm{an}}^{++}=A^{(+4)} .
$$

In contrast, in the $M$-formulation the effective current $L_{\mathrm{Mez}}^{++}$must obey the harmonic shortness condition (3.3) while the Grassmann analyticity constraint may be deformed

$$
\mathcal{D}^{++} L_{\mathrm{Mez}}^{++}=0, \quad \mathcal{D}_{\alpha}^{+} L_{\mathrm{Mez}}^{++}=A_{\alpha}^{(+3)} .
$$

Here $A^{(+4)}$ and $A_{\alpha}^{(+3)}$ are some composite operators of the vector multiplet which, in the non-abelian case, must obey the Wess-Zumino consistency condition [1] (see the next subsection). 
Let us denote the difference between $L_{\text {an }}^{++}$and $L_{\mathrm{Mez}}^{++}$by $\tilde{L}^{++}$,

$$
\tilde{L}^{++}=L_{\mathrm{an}}^{++}-L_{\mathrm{Mez}}^{++} .
$$

By construction, this superfield obeys

$$
\mathcal{D}^{++} \tilde{L}^{++}=A^{(+4)}, \quad \mathcal{D}_{\alpha}^{+} \tilde{L}^{++}=-A_{\alpha}^{(+3)} .
$$

Thus, given the superfield $\tilde{L}^{++}$, one could transfer the chiral anomaly from one formulation to the other.

According to the results of the previous section, see eq. (2.29), the admissible deformation of analyticity of the effective current reads

$$
A_{\alpha}^{(+3)}=\mathrm{i} \kappa \varepsilon_{\alpha \beta \gamma \delta} W^{+\beta} W^{+\gamma} W^{+\delta} .
$$

One of the aims of this work is to derive this expression for the chiral anomaly by analysing the short distance behaviour of the hypermultiplet propagator. The explicit form of $A^{(+4)}$ will be be discussed in section 5 .

\subsection{Consistent chiral anomaly in harmonic superspace}

In this subsection we make use of the $M$-formulation in which the variation of the effective action is given by the full-superspace integral (3.25), and the chiral anomaly appears as a deformation of the Grassmann analyticity constraint for the effective current (3.29). Since the $V$-formulation will not be discussed in this subsection, we omit the subscript 'Mez' assuming that we always work with the $M$-formulation, $L_{\mathrm{Mez}}^{++} \equiv L^{++}$.

Let us consider the variation of the effective action (3.25), where $\delta M^{--}$is the $\xi$-gauge transformation (3.7). The Wess-Zumino consistency condition [1] for this variation implies

$$
\left(\delta_{\xi_{1}} \delta_{\xi_{2}}-\delta_{\xi_{2}} \delta_{\xi_{1}}\right) \Gamma=\delta_{\left[\xi_{1}, \xi_{2}\right]} \Gamma
$$

where $\xi_{1}$ and $\xi_{2}$ are two gauge parameters taking values in the Lie algebra of the gauge group. In the non-abelian case the Wess-Zumino consistency condition (3.33) becomes a non-trivial constraint for the effective action which may, in principle, be solved using the descent equation approach [7]. In this section, however, to construct the consistent chiral anomaly we will follow the ideas of Leutwyler [9] generalised to the superfield formalism. Note that Leutwyler's approach has proved to be very efficient for obtaining the consistent anomalies of $4 \mathrm{D} \mathcal{N}=1$ supersymmetric gauge theories in superspace [24, 25].

Let $T^{\mathcal{A}}$ be the generators of the gauge group. The prepotential $M^{--}$may be written as a linear combination of the generators,

$$
M^{--}(z, u)=M_{\mathcal{A}}^{--}(z, u) T^{\mathcal{A}}
$$

Then, the variation (3.25) can be cast in the form

$$
\delta \Gamma\left[M^{--}\right]=\int \mathrm{d}^{6 \mid 8} z \mathrm{~d} u \delta M_{\mathcal{A}}^{--} L^{++\mathcal{A}}\left(M^{--}\right),
$$


where

$$
L^{++\mathcal{A}}=\operatorname{tr}_{(\mathrm{F})}\left(T^{\mathcal{A}} L^{++}\right) .
$$

Here the effective current $L^{++}$is treated as a function of the prepotential $M^{--}$(possibly, with superspace derivatives). The variation (3.35) is integrable provided that the effective current obeys

$$
\frac{\delta L^{++\mathcal{B}}\left(z_{2}, u_{2}\right)}{\delta M_{\mathcal{A}}^{--}\left(z_{1}, u_{1}\right)}-\frac{\delta L^{++\mathcal{A}}\left(z_{1}, u_{1}\right)}{\delta M_{\mathcal{B}}^{--}\left(z_{2}, u_{2}\right)}=0 .
$$

For such an effective current the variation (3.35) may be formally integrated

$$
\Gamma\left[M^{--}\right]=\int \mathrm{d}^{6 \mid 8} z \mathrm{~d} u M_{\mathcal{A}}^{--} \int_{0}^{1} \mathrm{~d} y L^{++\mathcal{A}}\left(y M^{--}\right) .
$$

For anomalous gauge theories, however, direct quantum computations usually result in an effective current which fails to satisfy (3.37) in the non-abelian case. We denote such a current by $L_{\text {cov }}^{++}$, emphasising that $\mathcal{D}_{\alpha}^{+} L_{\text {cov }}^{++}=A_{\alpha}^{(+3)}$ is a gauge-covariant superfield. The corresponding part of the variation of effective action with this current reads

$$
\delta \Gamma_{\operatorname{cov}}\left[M^{--}\right]=\int \mathrm{d}^{6 \mid 8} z \mathrm{~d} u \delta M_{\mathcal{A}}^{--} L_{\operatorname{cov}}^{++\mathcal{A}}\left(M^{--}\right) .
$$

Given the effective current $L_{\text {cov }}^{++}$, one can still construct a functional $\Gamma\left[M^{--}\right]$by the rule (3.38),

$$
\Gamma\left[M^{--}\right]=\int \mathrm{d}^{6 \mid 8} z \mathrm{~d} u M_{\mathcal{A}}^{--} \int_{0}^{1} \mathrm{~d} y L_{\text {cov }}^{++\mathcal{A}}\left(y M^{--}\right) .
$$

The general variation of this functional differs from (3.39) by the consistency terms which we denote here by $\delta \Gamma_{\text {cons }}$,

$$
\delta \Gamma\left[M^{--}\right]=\delta \Gamma_{\operatorname{cov}}\left[M^{--}\right]+\delta \Gamma_{\text {cons }}\left[M^{--}\right],
$$

where

$$
\begin{aligned}
\delta \Gamma_{\text {cons }}\left[M^{--}\right] & =\int_{0}^{1} \mathrm{~d} y X(y) \\
X(y)=y \int \mathrm{d}^{6 \mid 8} z_{1} \mathrm{~d} u_{1} \mathrm{~d}^{6 \mid 8} z_{2} \mathrm{~d} u_{2} \delta M_{\mathcal{A}}^{--}\left(z_{1}, u_{1}\right) M_{\mathcal{B}}^{--}\left(z_{2}, u_{2}\right) & \\
\times & {\left[\frac{\delta L_{\operatorname{cov}}^{++\mathcal{B}}\left(y M^{--}\left(z_{2}, u_{2}\right)\right)}{\delta\left(y M_{\mathcal{A}}^{--}\left(z_{1}, u_{1}\right)\right)}-\frac{\delta L_{\operatorname{cov}}^{++\mathcal{A}}\left(y M^{--}\left(z_{1}, u_{1}\right)\right)}{\delta\left(y M_{\mathcal{B}}^{--}\left(z_{2}, u_{2}\right)\right)}\right] . }
\end{aligned}
$$

Obviously, the variation (3.41) is integrable as it is derived from the functional (3.40). Thus, given an effective current $L_{\text {cov }}^{++}$which does not satisfy the consistency condition (3.37), it is always possible to construct the consistency terms (3.42) such that the variation (3.41) becomes integrable.

The relation between the analytic and Mezincescu's prepotentials (3.6) implies the variation rule

$$
\frac{\delta V_{\mathcal{A}}^{++}\left(z_{1}, u_{1}\right)}{\delta M_{\mathcal{B}}^{--}\left(z_{2}, u_{2}\right)}=\delta_{\mathcal{A}}^{\mathcal{B}} \delta_{\mathrm{A}}^{(2,2)}\left(\zeta_{1}, \zeta_{2}\right) \equiv \delta_{\mathcal{A}}^{\mathcal{B}}\left(\mathcal{D}^{+}\right)^{4} \delta^{6 \mid 8}\left(z_{1}-z_{2}\right) \delta^{(-2,2)}\left(u_{1}, u_{2}\right)
$$


This rule may be used to prove the useful identity

$$
\frac{\delta L^{++\mathcal{B}}\left(z_{2}, u_{2}\right)}{\delta M_{\mathcal{A}}^{--}\left(z_{1}, u_{1}\right)}=\int d \zeta_{3}^{(-4)} \frac{\delta V_{\mathcal{C}}^{++}\left(z_{3}, u_{3}\right)}{\delta M_{\mathcal{A}}^{--}\left(z_{1}, u_{1}\right)} \frac{\delta L^{++\mathcal{B}}\left(z_{2}, u_{2}\right)}{\delta V_{\mathcal{C}}^{++}\left(z_{3}, u_{3}\right)}=\frac{\delta L^{++\mathcal{B}}\left(z_{2}, u_{2}\right)}{\delta V_{\mathcal{A}}^{++}\left(z_{1}, u_{1}\right)}
$$

This identity allows us to represent the consistency terms (3.42b) in the equivalent form

$$
\begin{aligned}
X(y)=y \int \mathrm{d}^{6 \mid 8} & z_{1} \mathrm{~d} u_{1} \mathrm{~d}^{6 \mid 8} z_{2} \mathrm{~d} u_{2} \delta M_{\mathcal{A}}^{--}\left(z_{1}, u_{1}\right) M_{\mathcal{B}}^{--}\left(z_{2}, u_{2}\right) \\
& \times\left[\frac{\delta L_{\operatorname{cov}}^{++\mathcal{B}}\left(y V^{++}\left(z_{2}, u_{2}\right)\right)}{\delta\left(y V_{\mathcal{A}}^{++}\left(z_{1}, u_{1}\right)\right)}-\frac{\delta L_{\operatorname{cov}}^{++\mathcal{A}}\left(y V^{++}\left(z_{1}, u_{1}\right)\right)}{\delta\left(y V_{\mathcal{B}}^{++}\left(z_{2}, u_{2}\right)\right)}\right] .
\end{aligned}
$$

Here we consider the effective current as a function of the analytic prepotential $V^{++}$rather than the generalised Mezincescu prepotential $M^{--}$.

In this subsection, we have so far been considering the general variation $\delta M^{--}$. The consistent chiral anomaly appears when the $\xi$-gauge variation (3.7) is substituted in (3.41). In particular, eq. (3.39) turns into

$$
\delta_{\xi} \Gamma_{\text {cov }}=\int \mathrm{d}^{6 \mid 8} z \mathrm{~d} u \xi_{\mathcal{A}}^{(-3) \alpha} A_{\alpha}^{(+3) \mathcal{A}}
$$

where

$$
A_{\alpha}^{(+3)}=\mathcal{D}_{\alpha}^{+} L_{\mathrm{cov}}^{++}
$$

and $\xi^{(-3) \alpha}=\xi_{\mathcal{A}}^{(-3) \alpha} T^{\mathcal{A}}$. The consistency terms $\delta_{\xi} \Gamma_{\text {cons }}$ have the form (3.42a) with

$$
X(y)=-y \int \mathrm{d}^{6 \mid 8} z_{1} \mathrm{~d} u_{1} \mathrm{~d}^{6 \mid 8} z_{2} \mathrm{~d} u_{2} \xi_{\mathcal{A}}^{(-3) \alpha}\left(z_{1}, u_{1}\right) M_{\mathcal{B}}^{--}\left(z_{2}, u_{2}\right) \frac{\delta A_{\alpha}^{(+3) \mathcal{A}}\left(y V^{++}\left(z_{1}, u_{1}\right)\right)}{\delta\left(y V_{\mathcal{B}}^{++}\left(z_{2}, u_{2}\right)\right)}
$$

The relation (3.48) follows from (3.45) upon substituting the $\xi$-gauge variation (3.7), integrating the derivative $\mathcal{D}_{\alpha}^{+}$by parts and taking into account that only the last term in brackets in (3.45) may contribute since the other term is analytic in $\left(z_{1}, u_{1}\right)$ by construction. The equation (3.47) has also been applied.

We emphasise that in this section we discussed the properties of chiral anomaly for general $\mathcal{N}=(1,0)$ supersymmetric gauge theories in harmonic superspace. In the next section we will explicitly compute the covariant anomaly $A_{\alpha}^{(+3)}$ and the corresponding consistency terms for the model of a hypermultiplet interacting with an external vector multiplet.

\section{Chiral anomaly for the hypermultiplet effective action}

In this section we analyse the short-distance behaviour of the propagator for the hypermultiplet coupled to a background vector multiplet, and then apply the results of this analysis to compute the covariant and consistent chiral anomalies. 


\subsection{Hypermultiplet effective action}

In harmonic superspace, the hypermultiplet is described by an analytic superfield $q^{+}$, $\mathcal{D}_{\alpha}^{+} q^{+}=0$, and its tilde-conjugate $\tilde{q}^{+}$. The classical action of the hypermultiplet interacting with the gauge superfield $V^{++}$has the standard form $[42,45]$

$$
S=-\int \mathrm{d} \zeta^{(-4)} \tilde{q}^{+} \mathcal{D}^{++} q^{+}, \quad \mathcal{D}^{++}=D^{++}+\mathrm{i} V^{++} .
$$

This action is invariant under infinitesimal gauge transformations (3.2) with hypermultiplets transforming as

$$
\delta q^{+}=\mathrm{i} \lambda q^{+}, \quad \delta \tilde{q}^{+}=-\mathrm{i} \tilde{q}^{+} \lambda,
$$

where $\lambda$ is analytic gauge parameter. The hypermultiplet is assumed to transform in some representation of the gauge group.

The effective action in the hypermultiplet model (4.1) is given by

$$
\Gamma=\mathrm{i} \operatorname{Tr} \ln \mathcal{D}^{++}=-\mathrm{i} \operatorname{Tr} \ln G^{(1,1)},
$$

where the functional trace ' $\mathrm{Tr}$ ' corresponds to the space of analytic superfields of $\mathrm{U}(1)$ charge +1 , and $G^{(1,1)}$ is the hypermultiplet propagator obeying the equation

$$
\mathcal{D}_{1}^{++} G^{(1,1)}(1 \mid 2)=\delta_{\mathrm{A}}^{(3,1)}(1 \mid 2) \mathbb{1} .
$$

Here $\mathbb{1}$ is the unit matrix, and $\delta_{\mathrm{A}}^{(3,1)}(1 \mid 2)$ is the analytic delta-function which is related to the full superspace delta-function $\delta^{6 \mid 8}\left(z_{1}-z_{2}\right)$ as

$$
\delta_{\mathrm{A}}^{(q, 4-q)}(1 \mid 2)=\left(\mathcal{D}_{1}^{+}\right)^{4} \delta^{6 \mid 8}\left(z_{1}-z_{2}\right) \delta^{(q-4,4-q)}\left(u_{1}, u_{2}\right), \quad\left(\mathcal{D}^{+}\right)^{4}:=-\frac{1}{96} \varepsilon^{\alpha \beta \gamma \delta} \mathcal{D}_{\alpha}^{+} \mathcal{D}_{\beta}^{+} \mathcal{D}_{\gamma}^{+} \mathcal{D}_{\delta}^{+}
$$

The solution to (4.4) is derived in complete analogy with the $4 \mathrm{D} \mathcal{N}=2$ and $5 \mathrm{D} \mathcal{N}=1$ cases $[48,52]$. The solution $[36]$ is

$$
G^{(1,1)}(1 \mid 2)=\frac{1}{\widehat{\square}}\left(\mathcal{D}_{1}^{+}\right)^{4}\left(\mathcal{D}_{2}^{+}\right)^{4} \frac{\delta^{6 \mid 8}\left(z_{1}-z_{2}\right)}{\left(u_{1}^{+} u_{2}^{+}\right)^{3}} \mathbb{1},
$$

where $\widehat{\square}$ is the gauge covariant d'Alembertian operator which maps the space of covariantly analytic superfields into itself,

$$
\widehat{\square}=\frac{1}{2}\left(\mathcal{D}^{+}\right)^{4} \mathcal{D}^{--} \mathcal{D}^{--}
$$

Acting on an analytic superfield $\Phi_{\mathrm{A}}, \mathcal{D}_{\alpha}^{+} \Phi_{\mathrm{A}}=0$, it can equivalently be written as [36]

$$
\widehat{\square} \Phi_{\mathrm{A}}=\left(\mathcal{D}^{a} \mathcal{D}_{a}-W^{+\alpha} \mathcal{D}_{\alpha}^{-}+\frac{1}{4}\left(\mathcal{D}_{\alpha}^{-} W^{+\alpha}\right)-\frac{1}{4}\left(\mathcal{D}_{\alpha}^{+} W^{+\alpha}\right) \mathcal{D}^{--}\right) \Phi_{\mathrm{A}} .
$$

It is instructive to compare this expression for the $6 \mathrm{D} \mathcal{N}=(1,0)$ analytic d'Alembertian $\widehat{\square}$ with its $4 \mathrm{D} \mathcal{N}=2$ and $5 \mathrm{D} \mathcal{N}=1$ cousins $[48,56]$. 
The definition of the effective action (4.3) is purely formal, since the operator $\mathcal{D}^{++}$ maps the space of covariantly analytic superfields of $U(1)$ charge +1 into the space of covariantly analytic superfields of $\mathrm{U}(1)$ charge +3 . However, the variation of the effective action

$$
\delta \Gamma=-\operatorname{Tr}\left\{\delta V^{++} G^{(1,1)}\right\}
$$

makes sense. Explicitly, it can be written in the form

$$
\delta \Gamma=\operatorname{tr}_{(\mathrm{F})} \int \mathrm{d} \zeta^{(-4)} \delta V^{++} L_{\mathrm{an}}^{++},
$$

where the anomalous current supermultiplet $L_{\mathrm{an}}^{++}$is given by

$$
L_{\text {an }}^{++}:=-\left.G^{(1,1)}(1 \mid 2)\right|_{1=2} .
$$

The definition (4.9) is completely analogous to those in the $4 \mathrm{D} \mathcal{N}=2[51]$ and $5 \mathrm{D} \mathcal{N}=1$ cases [52]. The specific feature of the $6 \mathrm{D}$ case is that the variation (4.9) is not integrable due to chiral anomalies.

We point out that the effective current $(4.10 \mathrm{~b})$ is analytic owing to the analyticity of Green's function (4.6) with respect to both arguments. Starting from this expression for the propagator, it is possible to construct the effective current $L_{\mathrm{Mez}}^{++}$which is not analytic but satisfies the harmonic shortness constraint (3.29). For this purpose we revisit the form of the propagator in harmonic superspace developed in [51].

Recall that in the $\tau$-frame the covariant spinor derivative $\mathcal{D}_{\alpha}^{+}$is linear in harmonic variables, $\mathcal{D}_{\alpha}^{+}=\mathcal{D}_{\alpha}^{i} u_{i}^{+}$. For this derivative one can prove a simple identity

$$
\mathcal{D}_{2 \alpha}^{+} \delta^{6 \mid 8}\left(z_{1}-z_{2}\right)=\left[-\left(u_{1}^{+} u_{2}^{+}\right) \mathcal{D}_{1 \alpha}^{-}+\left(u_{1}^{-} u_{2}^{+}\right) \mathcal{D}_{1 \alpha}^{+}\right] \delta^{6 \mid 8}\left(z_{1}-z_{2}\right) .
$$

This identity, together with the algebra of gauge covariant derivatives (A.3), allows one to derive the useful property

$$
\begin{aligned}
\left(\mathcal{D}_{1}^{+}\right)^{4}\left(\mathcal{D}_{2}^{+}\right)^{4} & \frac{\delta^{6 \mid 8}\left(z_{1}-z_{2}\right)}{\left(u_{1}^{+} u_{2}^{+}\right)^{q}} \mathbb{1} \\
=\left(\mathcal{D}_{1}^{+}\right)^{4}\left[\frac{1}{\left(u_{1}^{+} u_{2}^{+}\right)^{q-4}}\left(\mathcal{D}_{1}^{-}\right)^{4}+\frac{\left(u_{1}^{-} u_{2}^{+}\right)}{\left(u_{1}^{+} u_{2}^{+}\right)^{q-3}} \Delta_{1}^{--}\right. & \\
& \left.\quad \widehat{\square}_{1} \frac{\left(u_{1}^{-} u_{2}^{+}\right)^{2}}{\left(u_{1}^{+} u_{2}^{+}\right)^{q-2}}+\frac{3-q}{4} \frac{\left(u_{1}^{-} u_{2}^{+}\right)^{3}}{\left(u_{1}^{+} u_{2}^{+}\right)^{q-1}}\left(\mathcal{D}_{\alpha}^{+} W^{+\alpha}\right)\right] \delta^{6 \mid 8}\left(z_{1}-z_{2}\right) \mathbb{1},
\end{aligned}
$$

where

$$
\Delta^{--}=-\frac{\mathrm{i}}{4}\left(\tilde{\gamma}^{a}\right)^{\alpha \beta} \mathcal{D}_{a} \mathcal{D}_{\alpha}^{-} \mathcal{D}_{\beta}^{-}-W^{-\alpha} \mathcal{D}_{\alpha}^{-}+\frac{1}{4}\left(\mathcal{D}_{\alpha}^{-} W^{-\alpha}\right) .
$$

Setting $q=3$ in (4.12), we get the following equivalent form for the hypermultiplet propagator (4.6)

$$
\begin{aligned}
G^{(1,1)}(1 \mid 2)=-\int_{0}^{\infty} \mathrm{d}(\mathrm{i} s)(\mathrm{i} s)^{\epsilon} \mathrm{e}^{\mathrm{i} s \widehat{\square}_{1}}\left(\mathcal{D}_{1}^{+}\right)^{4}\left[\left(\mathcal{D}_{1}^{-}\right)^{4}\left(u_{1}^{+} u_{2}^{+}\right)+\Delta_{1}^{--}\left(u_{1}^{-} u_{2}^{+}\right)\right. \\
\left.+\widehat{\square}_{1} \frac{\left(u_{1}^{-} u_{2}^{+}\right)^{2}}{\left(u_{1}^{+} u_{2}^{+}\right)}\right] \delta^{6 \mid 8}\left(z_{1}-z_{2}\right) \mathbb{1} .
\end{aligned}
$$


Here we have applied Schwinger's proper-time representation for the inverse d'Alembertian operator with $\epsilon$ being the ultraviolet regularisation parameter which should be set to zero at the end of computations. The expression (4.14) is manifestly covariantly analytic with respect to the first argument but the analyticity with respect to the other argument is implicit. It is an instructive exercise to check that (4.14) obeys $\mathcal{D}_{2 \alpha}^{+} G^{(1,1)}(1 \mid 2)=0$.

It is natural to represent (4.14) as a sum of two terms

$$
\begin{aligned}
& G^{(1,1)}(1 \mid 2)=G_{\text {reg }}^{(1,1)}(1 \mid 2)+G_{\text {sing }}^{(1,1)}(1 \mid 2), \\
& G_{\text {reg }}^{(1,1)}(1 \mid 2)=-\int_{0}^{\infty} \mathrm{d}(\mathrm{i} s)(\mathrm{i} s)^{\epsilon} \mathrm{e}^{\mathrm{i} s \widehat{\triangle}_{1}}\left(\mathcal{D}_{1}^{+}\right)^{4}\left[\left(\mathcal{D}_{1}^{-}\right)^{4}\left(u_{1}^{+} u_{2}^{+}\right)+\Delta_{1}^{--}\left(u_{1}^{-} u_{2}^{+}\right)\right] \delta^{6 \mid 8}\left(z_{1}-z_{2}\right) \mathbb{1}, \\
& G_{\text {sing }}^{(1,1)}(1 \mid 2)=-\int_{0}^{\infty} \mathrm{d}(\mathrm{i} s)(\mathrm{i} s)^{\epsilon} \mathrm{e}^{\mathrm{i} s \widehat{\square}_{1}}\left(\mathcal{D}_{1}^{+}\right)^{4} \widehat{\square}_{1} \frac{\left(u_{1}^{-} u_{2}^{+}\right)^{2}}{\left(u_{1}^{+} u_{2}^{+}\right)} \delta^{6 \mid 8}\left(z_{1}-z_{2}\right) \mathbb{1} .
\end{aligned}
$$

The idea of this splitting is that, at coincident superspace points, $G_{\text {reg }}^{(1,1)}$ can have UV quantum divergences, but has no singularity in the harmonic distribution. In contrast, $G_{\text {sing }}^{(1,1)}$ contains a harmonic singularity at coincident superspace points which is potentially dangerous, since there is no unambiguous procedure for regularising such divergencies. For the $4 \mathrm{D} \mathcal{N}=2$ and $5 \mathrm{D} \mathcal{N}=1$ hypermultiplet models, it was shown in [51, 52] that these harmonic singularities are not dangerous because all contributions to the effective action from the term like (4.15c) are vanishing owing to properties of the analytic delta-function at coincident superspace points. However, we will show below that the $6 \mathrm{D}$ distribution $(4.15 \mathrm{c})$ does give non-vanishing contributions to the effective action which are non-analytic and which correspond to the chiral anomaly.

The two parts of the hypermultiplet propagator (4.15b) and (4.15c) obey the following differential equations for $\epsilon=0$

$$
\begin{aligned}
& \mathcal{D}_{1}^{++} G_{\text {reg }}^{(1,1)}(1 \mid 2)=-2\left(u_{1}^{-} u_{2}^{+}\right)\left(\mathcal{D}_{1}^{+}\right)^{4} \delta^{6 \mid 8}\left(z_{1}-z_{2}\right), \\
& \mathcal{D}_{2}^{++} G_{\text {reg }}^{(1,1)}(1 \mid 2)=0, \\
& \mathcal{D}_{1}^{++} G_{\text {sing }}^{(1,1)}(1 \mid 2)=\delta_{\mathrm{A}}^{(3,1)}(1 \mid 2)+2\left(u_{1}^{-} u_{2}^{+}\right)\left(\mathcal{D}_{1}^{+}\right)^{4} \delta^{6 \mid 8}\left(z_{1}-z_{2}\right), \\
& \mathcal{D}_{2}^{++} G_{\text {sing }}^{(1,1)}(1 \mid 2)=-\delta_{\mathrm{A}}^{(1,3)}(1 \mid 2) .
\end{aligned}
$$

The expression in the right-hand side of (4.16a) contains no harmonic singularity and, thus, (4.16a) vanishes at coincident superspace points because of insufficient number of Grassmann derivatives acting on the superspace delta-function. The expressions (4.16c) and (4.16d), in contrast, contain harmonic singularities due to the harmonic delta-functions, see (4.5). These harmonic singularities require a regularisation which may make the expressions (4.16c) and (4.16d) non-trivial at coincident points. Such singular terms should be removed from the propagator in order to properly define the effective current $L_{\mathrm{Mez}}^{++}$ which has to obey the harmonic shortness constraint (3.29). Therefore, comparing (4.16) with (3.28) and (3.29), we conclude that at coincident superspace points $G_{\text {reg }}^{(1,1)}$ is responsible for the effective current $L_{\mathrm{Mez}}^{++}$,

$$
L_{\mathrm{Mez}}^{++}=-\left.G_{\mathrm{reg}}^{(1,1)}(1 \mid 2)\right|_{1=2},
$$


while $G_{\text {sing }}^{(1,1)}$ generates $\tilde{L}^{++}$,

$$
\tilde{L}^{++}=-\left.G_{\text {sing }}^{(1,1)}(1 \mid 2)\right|_{1=2} .
$$

While (4.17a) can be seen to contain no harmonic singularities, a harmonic regularisation is still required to give meaning to $(4.17 \mathrm{~b})$.

According to eqs. (3.28) and (3.29), the anomalies $A^{(+4)}$ and $A_{\alpha}^{(+3)}$ corresponding to the $V$ - and $M$-formulations of the theory are defined by the following formal expressions

$$
\begin{aligned}
& A^{(+4)}=-\mathcal{D}^{++}\left[\left.G^{(1,1)}(1 \mid 2)\right|_{1=2}\right]=-\mathcal{D}^{++}\left[\left.G_{\text {sing }}^{(1,1)}(1 \mid 2)\right|_{1=2}\right], \\
& A_{\alpha}^{(+3)}=-D_{\alpha}^{+}\left[\left.G_{\text {reg }}^{(1,1)}(1 \mid 2)\right|_{1=2}\right]=D_{\alpha}^{+}\left[\left.G_{\text {sing }}^{(1,1)}(1 \mid 2)\right|_{1=2}\right] .
\end{aligned}
$$

Below, we compute the chiral anomaly $A_{\alpha}^{(+3)}$ by analysing the short distance behaviour of the hypermultiplet propagator. The structure of $A^{(+4)}$ in the abelian case will be discussed in section 5 .

\subsection{Covariant chiral anomaly}

According to (4.18b), the chiral anomaly in Mezincescu's formulation of the gauge theory is defined by the part of the hypermultiplet propagator $(4.15 \mathrm{c})$. Using the identity $\left(\mathcal{D}^{+}\right)^{4} \widehat{\square}=\widehat{\square}\left(\mathcal{D}^{+}\right)^{4}$ the latter may be represented in the equivalent form

$$
G_{\text {sing }}^{(1,1)}(1 \mid 2)=\epsilon \int_{0}^{\infty} \mathrm{d}(\mathrm{i} s)(\mathrm{i} s)^{\epsilon-1} \mathrm{e}^{\mathrm{i} s \widehat{\square}_{1}} \frac{\left(u_{1}^{-} u_{2}^{+}\right)^{2}}{\left(u_{1}^{+} u_{2}^{+}\right)}\left(\mathcal{D}_{1}^{+}\right)^{4} \delta^{6 \mid 8}\left(z_{1}-z_{2}\right) \mathbb{1} .
$$

Note that this expression is analytic only in the first argument. Hence, eq. (4.18b) implies

$$
\begin{aligned}
A_{\alpha}^{(+3)} & =\left.\mathcal{D}_{2 \alpha}^{+} G_{\text {sing }}^{(1,1)}(1 \mid 2)\right|_{1=2} \\
& =-\left.\epsilon \int_{0}^{\infty} \mathrm{d}(\mathrm{i} s)(\mathrm{i} s)^{\epsilon-1} \mathrm{e}^{\mathrm{i} s \widehat{\square}_{1}}\left(u_{1}^{-} u_{2}^{+}\right)^{2}\left(\mathcal{D}_{1}^{+}\right)^{4} \mathcal{D}_{1 \alpha}^{-} \delta^{6 \mid 8}\left(z_{1}-z_{2}\right) \mathbb{1}\right|_{1=2},
\end{aligned}
$$

where we applied the identity (4.11) to achieve the last line.

In (4.20), one has to expand the exponent of the operator (4.8) in a series and accumulate eight Grassmann derivatives to apply the identity

$$
\left.\left(D^{+}\right)^{4}\left(D^{-}\right)^{4} \delta^{8}\left(\theta_{1}-\theta_{2}\right)\right|_{1=2}=1 .
$$

It is sufficient to consider a covariantly constant on-shell vector multiplet

$$
\mathcal{D}_{a} W^{+\alpha}=0, \quad \mathcal{D}_{\alpha}^{+} W^{+\alpha}=\mathcal{D}_{\alpha}^{-} W^{+\alpha}=0 .
$$

For such a background, the form of the operator (4.8) simplifies:

$$
\widehat{\square}=\mathcal{D}^{a} \mathcal{D}_{a}-W^{+\alpha} \mathcal{D}_{\alpha}^{-} .
$$

Taking this into account, from (4.20) we find

$$
\begin{aligned}
A_{\alpha}^{(+3)} & =-\left.\frac{2}{3} \epsilon \int_{0}^{\infty} \mathrm{d}(\mathrm{i} s)(\mathrm{i} s)^{\epsilon+2} \varepsilon_{\alpha \beta \gamma \delta} W^{+\beta} W^{+\gamma} W^{+\delta} \mathrm{e}^{\mathrm{i} s \mathcal{D}^{a} \mathcal{D}_{a}} \delta^{6}\left(x_{1}-x_{2}\right) \mathbb{1}\right|_{1=2} \\
& =-\frac{\mathrm{i}}{96 \pi^{3}} \varepsilon_{\alpha \beta \gamma \delta} W^{+\beta} W^{+\gamma} W^{+\delta} \epsilon \int_{0}^{\infty} \mathrm{d}(\mathrm{i} s)(\mathrm{i} s)^{\epsilon-1}
\end{aligned}
$$


Here, in the last line, we applied the identity

$$
\left.\mathrm{e}^{\mathrm{i} \epsilon \mathcal{D}^{a} \mathcal{D}_{a}} \delta^{6}\left(x_{1}-x_{2}\right) \mathbb{1}\right|_{x_{1}=x_{2}}=\int \frac{\mathrm{d}^{6} k}{(2 \pi)^{6}} \mathrm{e}^{\mathrm{i} \epsilon\left(\mathcal{D}^{a}+\mathrm{i} k^{a}\right)\left(\mathcal{D}_{a}+\mathrm{i} k_{a}\right)} \mathbb{1}=-\frac{\mathbb{1}}{64 \pi^{3} \epsilon^{3}}+O\left(\epsilon^{-2}\right),
$$

where the terms $O\left(\epsilon^{-2}\right)$ are irrelevant for small $\epsilon$.

The integration over the proper time always assumes the exponent $\mathrm{e}^{-\alpha s}, \alpha>0$, in the integrand which makes the integral convergent. In the limit of small $\epsilon$, the identity

$$
\lim _{\epsilon \rightarrow 0} \epsilon \int_{0}^{\infty} \mathrm{d}(\mathrm{i} s)(\mathrm{i} s)^{\epsilon-1} \mathrm{e}^{-\alpha s}=1
$$

yields

$$
A_{\alpha}^{(+3)}=-\frac{\mathrm{i}}{96 \pi^{3}} \varepsilon_{\alpha \beta \gamma \delta} W^{+\beta} W^{+\gamma} W^{+\delta} .
$$

The Lie-algebra valued field strength $W^{+\alpha}$ and gauge parameter $\xi^{(-3) \alpha}$ may be written as linear combinations of the generators $T^{\mathcal{A}}$ of the gauge group,

$$
W^{+\alpha}=W_{\mathcal{A}}^{+\alpha} T^{\mathcal{A}}, \quad \xi^{(-3) \alpha}=\xi_{\mathcal{A}}^{(-3) \alpha} T^{\mathcal{A}} .
$$

In terms of the superfields $W_{\mathcal{A}}^{+\alpha}$, the anomaly (4.27) reads

$$
A_{\alpha}^{(+3) \mathcal{A}}=\operatorname{str}\left(T^{\mathcal{A}} A_{\alpha}^{(+3)}\right)=-\frac{\mathrm{i}}{96 \pi^{3}} d^{\mathcal{A B C D}} \varepsilon_{\alpha \beta \gamma \delta} W_{\mathcal{B}}^{+\beta} W_{\mathcal{C}}^{+\gamma} W_{\mathcal{D}}^{+\delta},
$$

where $d^{\mathcal{A B C D}}$ is the gauge-invariant tensor (2.26). Comparing (4.29) with (2.29) we find the value of the coefficient $\kappa$ for the model of hypermultiplet in external gauge superfield

$$
\kappa=-\frac{1}{96 \pi^{3}} .
$$

The covariant chiral anomaly manifests itself as a deformation of the analyticity of the effective current (3.29) which is expressed through the part of the hypermultiplet propagator $(4.16 \mathrm{~d})$ with harmonic singularities at coincident points. In the four-dimensional case, it was proved in [51] that all contributions to the hypermultiplet effective action from such a term are vanishing, and the analyticity of the effective current is preserved. In this paper, we demonstrate that in the six-dimensional case this term in the hypermultiplet propagator plays an important role since it generates the anomalous part of the hypermultiplet effective action.

\subsection{Consistency terms}

The procedure for constructing the consistent chiral anomaly in harmonic superspace is described in section 3.3. The consistency terms are given by the non-local functional (3.48). Substituting the covariant chiral anomaly in the form (4.29) into this functional we get

$$
\begin{aligned}
X(y)= & \frac{\mathrm{i}}{32 \pi^{3}} d^{\mathcal{A B C D}} y \int \mathrm{d}^{6 \mid 8} z_{1} \mathrm{~d} u_{1} \mathrm{~d}^{6 \mid 8} z_{2} \mathrm{~d} u_{2} \xi_{\mathcal{A}}^{(-3) \alpha}\left(z_{1}, u_{1}\right) \varepsilon_{\alpha \beta \gamma \delta} M_{\mathcal{E}}^{--}\left(z_{2}, u_{2}\right) \\
& \times\left.\left(W_{\mathcal{B}}^{+\beta}\left(z_{1}, u_{1}\right) W_{\mathcal{C}}^{+\gamma}\left(z_{1}, u_{1}\right) \frac{\delta W_{\mathcal{D}}^{+\delta}\left(z_{1}, u_{1}\right)}{\delta V_{\mathcal{E}}^{++}\left(z_{2}, u_{2}\right)}\right)\right|_{V^{++} \rightarrow y V^{++}} .
\end{aligned}
$$


Note that the harmonic zero-curvature equation (A.12) implies the relation

$$
\frac{\delta V^{--}\left(z_{1}, u_{1}\right)}{\delta V^{++}\left(z_{2}, u_{2}\right)}=\mathrm{e}^{\mathrm{i} b\left(z_{1}, u_{1}\right)}\left(\mathcal{D}_{2}^{+}\right)^{4}\left[\mathrm{e}^{-\mathrm{i} b\left(z_{2}, u_{2}\right)} \frac{\delta^{6 \mid 8}\left(z_{1}-z_{2}\right)}{\left(u_{1}^{+} u_{2}^{+}\right)^{2}}\right],
$$

where $b(z, u)=b_{\mathcal{A}}(z, u) T^{\mathcal{A}}$ is the Lie-algebra-valued bridge superfield (see eq. (A.7)). Taking advantage of (4.32) and (A.15), the variational derivative of the superfield strength $W^{+\alpha}$ may be brought to the form

$$
\frac{\delta W_{\mathcal{D}}^{+\alpha}\left(z_{1}, u_{1}\right)}{\delta V_{\mathcal{E}}^{++}\left(z_{2}, u_{2}\right)}=\frac{\mathrm{i}}{24} \varepsilon^{\alpha \beta \gamma \delta} \mathcal{D}_{1 \beta}^{+} \mathcal{D}_{1 \gamma}^{+} \mathcal{D}_{1 \delta}^{+}\left(\mathcal{D}_{2}^{+}\right)^{4}\left[\mathrm{e}^{\mathrm{i} b\left(z_{1}, u_{1}\right)} \mathrm{e}^{-\mathrm{i} b\left(z_{2}, u_{2}\right)} \frac{\delta^{6 \mid 8}\left(z_{1}-z_{2}\right)}{\left(u_{1}^{+} u_{2}^{+}\right)^{2}}\right]_{\mathcal{D E}} .
$$

Substituting this variation into (4.31) and integrating over $z_{2}$ we get the final expression for the consistency terms

$$
\begin{aligned}
X(y)= & \frac{d^{\mathcal{A B C} \mathcal{B}}}{128 \pi^{3}} y \int \mathrm{d}^{6 \mid 8} z \mathrm{~d} u \mathrm{~d} u^{\prime} \frac{V_{\mathcal{E}}^{++}\left(z, u^{\prime}\right)}{\left(u^{+} u^{+\prime}\right)^{2}} \\
& \times\left.\left\{\mathcal{D}_{\alpha}^{+} \mathcal{D}_{\beta}^{+} \mathcal{D}_{\gamma}^{+}\left[\xi_{\mathcal{A}}^{(-3) \alpha} W_{\mathcal{B}}^{+\beta} W_{\mathcal{C}}^{+\gamma}\right]\left(\mathrm{e}^{\mathrm{i} b(z, u)} \mathrm{e}^{-\mathrm{i} b\left(z, u^{\prime}\right)}\right)_{\mathcal{D E}}\right\}\right|_{V^{++} \rightarrow y V^{++}} .
\end{aligned}
$$

At the end of this section we give the resulting expression for the consistent chiral anomaly in the $\mathcal{N}=(1,0)$ superspace, which is a sum of the covariant anomaly (3.46) with $A_{\alpha}^{(+3) \mathcal{A}}$ as in (4.29), and the consistency term (3.42a) with $X(y)$ given by (4.34)

$$
\begin{aligned}
\delta_{\xi} \Gamma= & -\frac{\mathrm{i}}{96 \pi^{3}} d^{\mathcal{A B C D}} \int \mathrm{d}^{6 \mid 8} z \mathrm{~d} u \xi_{\mathcal{A}}^{(-3) \alpha} \varepsilon_{\alpha \beta \gamma \delta} W_{\mathcal{B}}^{+\beta} W_{\mathcal{C}}^{+\gamma} W_{\mathcal{D}}^{+\delta} \\
& +\frac{d^{\mathcal{A B C} \mathcal{D}}}{128 \pi^{3}} \int_{0}^{1} \mathrm{~d} y y \int \mathrm{d}^{6 \mid 8} z \mathrm{~d} u \mathrm{~d} u^{\prime} \frac{V_{\mathcal{E}}^{++}\left(z, u^{\prime}\right)}{\left(u^{+} u^{+\prime}\right)^{2}} \\
& \times\left.\left\{\mathcal{D}_{\alpha}^{+} \mathcal{D}_{\beta}^{+} \mathcal{D}_{\gamma}^{+}\left[\xi_{\mathcal{A}}^{(-3) \alpha} W_{\mathcal{B}}^{+\beta} W_{\mathcal{C}}^{+\gamma}\right]\left(\mathrm{e}^{\mathrm{i} b(z, u)} \mathrm{e}^{-\mathrm{i} b\left(z, u^{\prime}\right)}\right)_{\mathcal{D E}}\right\}\right|_{V^{++} \rightarrow y V^{++}}
\end{aligned}
$$

An interesting feature of this expression is that it is local in the superspace coordinates $z^{A}=\left(x^{a}, \theta_{i}^{\alpha}\right)$, but is non-local in the harmonics $u$. This resembles the SYM classical action in the harmonic superspace which is also non-local in the harmonic variables [43].

\subsection{Abelian limit}

The field strength $W^{+\alpha}$ depends linearly on the harmonic connection $V^{--}$, in accordance with (A.15), while $V^{--}$is a non-linear function of the analytic prepotential $V^{++}$in the non-abelian case. The explicit expression for $V^{--}$in terms of $V^{++}$was given in [44]. Modulo a $\tau$-gauge transformation, the bridge superfield $b(z, u)$ is also a non-linear function of $V^{++}$which was presented in [57]. The formula (4.34) suggests that in all these nonlinear functions the analytic prepotential $V^{++}$should be replaced with $y V^{++}$, making $X(y)$ a highly non-trivial function of $y$. However, in the abelian case this dependence on $y$ simplifies such that the integration over $y$ may be easily done in (3.42a). Indeed, in the abelian case the variational derivative (4.33) reduces to

$$
\frac{\delta W^{+\alpha}\left(z_{1}, u_{1}\right)}{\delta V^{++}\left(z_{2}, u_{2}\right)}=\frac{\mathrm{i}}{24} \varepsilon^{\alpha \beta \gamma \delta} D_{1 \beta}^{+} D_{1 \gamma}^{+} D_{1 \delta}^{+}\left(D_{2}^{+}\right)^{4} \frac{\delta^{6 \mid 8}\left(z_{1}-z_{2}\right)}{\left(u_{1}^{+} u_{2}^{+}\right)^{2}}
$$


so that the consistency term (4.31) reads

$$
\begin{aligned}
X(y)= & \frac{1}{128 \pi^{3}} y^{3} \int \mathrm{d}^{6 \mid 8} z_{1} \mathrm{~d} u_{1} \mathrm{~d}^{6 \mid 8} z_{2} \mathrm{~d} u_{2} \xi^{(-3) \alpha}\left(z_{1}, u_{1}\right) W^{+\beta}\left(z_{1}, u_{1}\right) W^{+\gamma}\left(z_{1}, u_{1}\right) \\
& \times M^{--}\left(z_{2}, u_{2}\right) D_{1 \beta}^{+} D_{1 \gamma}^{+} D_{1 \delta}^{+}\left(D_{2}^{+}\right)^{4} \frac{\delta^{6 \mid 8}\left(z_{1}-z_{2}\right)}{\left(u_{1}^{+} u_{2}^{+}\right)^{2}} .
\end{aligned}
$$

Now we integrate the derivatives $\left(D_{2}^{+}\right)^{4}$ by parts and restore the analytic prepotential $V^{++}$from $M^{--}$using the identity (3.6). The analytic prepotential $V^{++}$is related to $V^{--}$ through the simple identity

$$
V^{--}(z, u)=\int \mathrm{d} u^{\prime} \frac{V^{++}\left(z, u^{\prime}\right)}{\left(u^{+} u^{+\prime}\right)^{2}}
$$

which is the solution of the zero-curvature equation (A.12) in the abelian case. Finally, the delta-function in (4.37) allows us to integrate over $\mathrm{d}^{6 \mid 8} z_{2}$, and the derivatives $D_{1 \beta}^{+} D_{1 \gamma}^{+} D_{1 \delta}^{+}$ convert $V^{--}$into the superfield strength $W^{+\alpha}$ according to (A.15),

$$
X(y)=\frac{\mathrm{i}}{32 \pi^{3}} y^{3} \int \mathrm{d}^{6 \mid 8} z \mathrm{~d} u \xi^{(-3) \alpha} \varepsilon_{\alpha \beta \gamma \delta} W^{+\beta} W^{+\gamma} W^{+\delta} .
$$

After integrating (4.39) over $\mathrm{d} y$ and adding the abelian version of (4.29), we get

$$
\delta_{\xi} \Gamma=-\frac{\mathrm{i}}{384 \pi^{3}} \int \mathrm{d}^{6 \mid 8} z \mathrm{~d} u \xi^{(-3) \alpha} \varepsilon_{\alpha \beta \gamma \delta} W^{+\beta} W^{+\gamma} W^{+\delta} .
$$

It shows that the consistent anomaly differs from the covariant anomaly (4.27) in the abelian limit by the factor $\frac{1}{4}$. This interplay between the coefficients in the covariant and consistent anomalies is the same as in the non-supersymmetric case $[7,8]$. This is a nontrivial check that the result (4.35) is the correct expression for consistent chiral anomaly in $(1,0)$ superspace.

\section{Concluding comments}

In this paper, the chiral anomalies in general $6 \mathrm{D} \mathcal{N}=(1,0)$ supersymmetric gauge theories realised in harmonic superspace have been computed. We started by recalling that there exist two different (but related) approaches to formulate such gauge theories which are based on the use of either the analytic gauge connection $V^{++}$or the generalised Mezincescu prepotential $M^{--}$. Since the gauge prepotentials $V^{++}$and $M^{--}$are different off-shell supermultiplets, the gauge transformations in the $V$ - and $M$-formulations are also different. They are described by eqs. (3.2) and (3.10), respectively. This difference implies that the chiral anomaly manifests itself quite differently in the two formulations: either as a deformation of the harmonic shortness constraint of the effective current (3.28) in the $V$-formulation or as a deformation of the Grassmann analyticity of the effective current, eq. (3.29), in the $M$-formulation. In the $M$-formulation, the covariant anomaly is given by eq. (4.27). We constructed consistency terms such that the full chiral anomaly 
obeys the Wess-Zumino consistency condition. The procedure of constructing these consistency terms is a generalisation of Leutwyler's ideas [9] to gauge theories in $6 \mathrm{D} \mathcal{N}=(1,0)$ harmonic superspace.

Our results remain valid for the higher-derivative $\mathcal{N}=(1,0)$ supersymmetric gauge theory constructed in [58]. At the component level, the chiral anomalies in this theory were discussed in [59].

In this section, we will discuss in some detail the issue of constructing the chiral anomaly in the $V$-formulation of gauge theory. In particular, we deduce an expression for the chiral anomaly $A^{(+4)}$ in the abelian case. To construct this anomaly, we will follow the procedure proposed in our recent work [30] which allows one to restore $A^{(+4)}$ when the expression for $A_{\alpha}^{(+3)}$ is known.

In the $M$-formulation, the gauge theory is described by the effective current $L_{\mathrm{Mez}}^{++}(z, u)=u_{i}^{+} u_{j}^{+} L_{\mathrm{Mez}}^{i j}(z)$ obeying

$$
D_{\alpha}^{+} L_{\mathrm{Mez}}^{++}=\mathrm{i} \kappa \varepsilon_{\alpha \beta \gamma \delta} W^{+\beta} W^{+\gamma} W^{+\delta},
$$

where $\kappa$ is given by (4.30) for the model of hypermultiplet interacting with background vector multiplet. Let us introduce a superfield $F^{++}(z, u)$ as a solution of the equation

$$
D_{\alpha}^{+} F^{++}=\mathrm{i} \kappa \varepsilon_{\alpha \beta \gamma \delta} W^{+\beta} W^{+\gamma} W^{+\delta}
$$

and defined modulo arbitrary shift of the form

$$
F^{++} \rightarrow F^{++}+H^{++}, \quad D_{\alpha}^{+} H^{++}=0 .
$$

A particular solution of $(5.2)$ is

$$
F^{++}=-\frac{\mathrm{i}}{2} \kappa V_{\alpha \beta} W^{+\alpha} W^{+\beta}-\frac{\mathrm{i}}{64} \kappa \varepsilon^{\alpha \beta \gamma \delta} V_{\alpha \beta} V_{\gamma \delta} D^{+} W^{+},
$$

where $V_{\alpha \beta}$ is the connection defined in (A.14b). This solution has the following important property

$$
D^{++} F^{++}=-\frac{\mathrm{i}}{2} \kappa G^{++\alpha \beta} \partial_{\alpha \beta} V^{++},
$$

where

$$
G^{++\alpha \beta}=W^{+\alpha} W^{+\beta}+\frac{1}{16} \varepsilon^{\alpha \beta \gamma \delta} V_{\gamma \delta} D^{+} W^{+}, \quad D_{\gamma}^{+} G^{++\alpha \beta}=0 .
$$

The property (5.5) shows that $D^{++} F^{++}$is analytic and, thus, may appear as a part of the anomaly superfield $A^{(+4)}$ in the $V$-formulation of the gauge theory.

Let us now introduce the following superfield

$$
\mathbb{L}^{++}=L_{\mathrm{Mez}}^{++}-F^{++},
$$

which is analytic due to the properties (5.1) and (5.2),

$$
D_{\alpha}^{+} \mathbb{L}^{++}=0
$$


However, unlike $L_{\mathrm{Mez}}^{++}$, this superfield is no longer holomorphic on $\mathbb{C} P^{1}$,

$$
D^{++} \mathbb{L}^{++}=\mathbb{A}^{(+4)}, \quad D_{\alpha}^{+} \mathbb{A}^{(+4)}=0 .
$$

Thus, the chiral anomaly is completely encoded in the analytic superfield $\mathbb{A}^{(+4)}$ which is defined modulo shifts

$$
\mathbb{A}^{(+4)} \rightarrow \mathbb{A}^{(+4)}-D^{++} H^{++},
$$

which follow from the freedom in the definition of $F^{++}$, see (5.3).

For the choice of $F^{++}$as in eq. (5.4), the superfield $\mathbb{A}^{(+4)}$ reads

$$
\mathbb{A}^{(+4)}=\frac{\mathrm{i}}{2} \kappa G^{++\alpha \beta} \partial_{\alpha \beta} V^{++} .
$$

However, this superfield is not yet the anomaly $A^{(+4)}$ which corresponds to the $V$ formulation of the gauge theory with effective current $L_{\text {an }}^{++}$obeying (3.28). The problem is that (5.11) does not satisfy the Wess-Zumino consistency condition. Indeed, it varies under the gauge transformation (3.2) as

$$
\delta_{\lambda} \mathbb{A}^{(+4)}=-\frac{\mathrm{i}}{2} \kappa D^{++}\left(G^{++\alpha \beta} \partial_{\alpha \beta} \lambda\right) .
$$

This implies that $\mathbb{A}^{(+4)}$ cannot appear as the gauge variation of an effective action, $\delta_{\lambda} \Gamma=$ $\int \mathrm{d} \zeta^{(-4)} \lambda \mathbb{A}^{(+4)}$, since $\delta_{\lambda_{1}} \delta_{\lambda_{2}} \Gamma \neq \delta_{\lambda_{2}} \delta_{\lambda_{1}} \Gamma$.

To resolve this problem, the arbitrariness (5.10) should be employed. Indeed, we propose the following non-local expression for $\mathrm{H}^{++}$

$$
\begin{aligned}
H^{++}(\zeta) & =-\int \mathrm{d} \zeta^{(-4)} G^{(2,0)}\left(\zeta, \zeta^{\prime}\right) \mathbb{B}^{(+4)}\left(\zeta^{\prime}\right), \\
\mathbb{B}^{(+4)} & =\frac{\mathrm{i}}{2} \kappa \partial_{\alpha \beta}\left(V^{++} G^{++\alpha \beta}\right),
\end{aligned}
$$

where $G^{(2,0)}\left(\zeta, \zeta^{\prime}\right)$ is Green's function

$$
G^{(2,0)}\left(\zeta, \zeta^{\prime}\right)=\frac{1}{\square}\left(D^{+}\right)^{4}\left(D^{+\prime}\right)^{4}\left[\delta^{6 \mid 8}\left(z-z^{\prime}\right) \frac{\left(u^{+} u^{-\prime}\right)}{\left(u^{+} u^{+\prime}\right)^{3}}\right]
$$

with the property

$$
D^{++} G^{(2,0)}\left(\zeta, \zeta^{\prime}\right)=\delta_{\mathrm{A}}^{(4,0)}\left(\zeta, \zeta^{\prime}\right) .
$$

The expression (5.13) is chosen such that the superfield

$$
\begin{aligned}
A^{(+4)} & =\mathbb{A}^{(+4)}-D^{++} H^{++}=\mathbb{A}^{(+4)}+\mathbb{B}^{(+4)} \\
& =\mathrm{i} \kappa G^{++\alpha \beta} \partial_{\alpha \beta} V^{++}+\frac{\mathrm{i}}{2} \kappa V^{++} \partial_{\alpha \beta} G^{++\alpha \beta}
\end{aligned}
$$

is analytic, $D_{\alpha}^{+} A^{(+4)}=0$, and obeys the Wess-Zumino consistency condition. Indeed, we can consistently associate with (5.16) an effective action $\Gamma$, since the variation defined by

$$
\delta_{\lambda} \Gamma=\int \mathrm{d} \zeta^{(-4)} \lambda A^{(+4)}
$$


is integrable,

$$
\left(\delta_{\lambda_{1}} \delta_{\lambda_{2}}-\delta_{\lambda_{2}} \delta_{\lambda_{1}}\right) \Gamma=0
$$

We stress that the integrability condition (5.18) is nontrivial already in the abelian case since the function (5.16) is not gauge invariant. This confirms that this function can consistently describe the chiral anomaly in the $V$-formulation of the gauge theory.

It is very tempting to derive $A^{(+4)}$ by direct supergraph computations in the harmonic superspace. ${ }^{6}$ Another important issue is to construct a generalisation of (5.16) to the case of non-abelian chiral anomaly. We leave these issues for future studies.

\section{Acknowledgments}

We are grateful to the referee for useful suggestions. We thank Ian McArthur for comments on the manuscript. IBS is grateful to Evgeny Buchbinder and Ian McArthur for useful discussions, and to the School of Physics and Astrophysics at UWA, where the major part of this work was done, for kind hospitality. IBS acknowledges the support from the RFBR grant No 15-02-06670. SMK and IBS were supported in part by the Australian Research Council, project No. DP140103925. The work of SMK is also supported in part by the Australian Research Council, project No. DP160103633. JN acknowledges support from GIF the German-Israeli Foundation for Scientific Research and Development.

\section{A Vector multiplet in harmonic superspace}

Supersymmetric Yang-Mills theory in $6 \mathrm{D} \mathcal{N}=(1,0)$ harmonic superspace was formulated in [42-44]. Here we briefly review this formulation following the harmonic superspace notation of [46].

Let $u_{i}^{+}$and $u_{i}^{-}$be standard $\mathrm{SU}(2)$ harmonic variables, $\left(u_{i}^{-}, u_{i}^{+}\right) \in \mathrm{SU}(2)$,

$$
\overline{u^{+i}}=u_{i}^{-}, \quad u^{+i} u_{i}^{-}=1,
$$

with $u_{i}^{+}=\varepsilon_{i j} u^{+j}$. Let $D^{++}, D^{--}$and $D^{0}$ be the associated harmonic derivatives defined as in [46]. Using the harmonics we introduce a new basis for the gauge-covariant spinor derivatives

$$
\mathcal{D}_{\alpha}^{ \pm}=u_{i}^{ \pm} \mathcal{D}_{\alpha}^{i}=D_{\alpha}^{ \pm}+\mathrm{i} V_{\alpha}^{ \pm}, \quad V_{\alpha}^{ \pm}=u_{i}^{ \pm} V_{\alpha}^{i}
$$

In accordance with (2.2) the operators (A.2) obey the following (anti)commutation relations

$$
\begin{aligned}
\left\{\mathcal{D}_{\alpha}^{+}, \mathcal{D}_{\beta}^{+}\right\} & =0, \\
\left\{\mathcal{D}_{\alpha}^{+}, \mathcal{D}_{\beta}^{-}\right\} & =2 \mathrm{i}\left(\gamma^{a}\right)_{\alpha \beta} \mathcal{D}_{a}, \\
{\left[\mathcal{D}_{a}, \mathcal{D}_{\alpha}^{ \pm}\right] } & =\mathrm{i}\left(\gamma_{a}\right)_{\alpha \beta} W^{ \pm \beta}, \\
{\left[\mathcal{D}_{a}, \mathcal{D}_{b}\right] } & =\mathrm{i} F_{a b},
\end{aligned}
$$

\footnotetext{
${ }^{6}$ It would be also very interesting to study the problem of computing the chiral anomalies in $\mathcal{N}=(1,0)$ supersymmetric gauge theories using the supergraph technique in projective superspace developed in [60] and references therein.
} 
where $W^{ \pm \alpha}$ are the irreducible $\mathrm{U}(1)$ components of the field strength $W^{i \alpha}$,

$$
W^{ \pm \alpha}=u_{i}^{ \pm} W^{i \alpha}
$$

In the harmonic superspace setting, it is useful to combine the superspace gaugecovariant derivatives with the harmonic ones,

$$
\mathcal{D}_{\hat{A}}=\left(\mathcal{D}_{a}, \mathcal{D}_{\alpha}^{ \pm}, \mathcal{D}^{++}, \mathcal{D}^{--}, \mathcal{D}^{0}\right):=\left(\mathcal{D}_{a}, \mathcal{D}_{\alpha}^{ \pm}, D^{++}, D^{--}, D^{0}\right)=D_{\hat{A}}+\mathrm{i} V_{\hat{A}}
$$

The gauge transformation of $\mathcal{D}_{\hat{A}}$ is analogous to (2.6),

$$
\mathcal{D}_{\hat{A}} \longrightarrow \mathcal{D}_{\hat{A}}^{\prime}=\mathrm{e}^{\mathrm{i} \tau} \mathcal{D}_{\hat{A}} \mathrm{e}^{-\mathrm{i} \tau} .
$$

Since the gauge superfield parameter $\tau$ is harmonic independent, the harmonic derivatives $\left(D^{ \pm \pm}, D^{0}\right)$ are gauge covariant.

The equation (A.3a) is the integrability condition for covariantly analytic superfields to exist. This equation can be solved in terms of a bridge superfield $b=b(z, u)$ defined by the rule

$$
\mathcal{D}_{\alpha}^{+}=\mathrm{e}^{-\mathrm{i} b} D_{\alpha}^{+} \mathrm{e}^{\mathrm{i} b} .
$$

The introduction of the bridge superfield leads to a new gauge freedom, in addition to the $\tau$-gauge transformations (2.6). The complete gauge transformation law of $b$ is

$$
\mathrm{e}^{\mathrm{i} b^{\prime}}=\mathrm{e}^{\mathrm{i} \lambda} \mathrm{e}^{\mathrm{i} b} \mathrm{e}^{-\mathrm{i} \tau}
$$

where $\lambda$ is a $\mathrm{U}(1)$ neutral analytic superfield, $D_{\alpha}^{+} \lambda=0$.

The representation (A.5) for the gauge-covariant derivatives is called the $\tau$-frame. When it is important, we will attach a label ' $(\tau)$ ' to the covariant derivatives in this representation, $\mathcal{D}_{(\tau) \hat{A}}$. Using the bridge superfield one can introduce another representation for these derivatives, which is usually referred to as the $\lambda$-frame,

$$
\mathcal{D}_{(\tau) \hat{A}} \longrightarrow \mathcal{D}_{(\lambda) \hat{A}}=\mathrm{e}^{\mathrm{i} b} \mathcal{D}_{(\tau) \hat{A}} \mathrm{e}^{-\mathrm{i} b}=D_{\hat{A}}+\mathrm{i} V_{(\lambda) \hat{A}}
$$

Below, we will consider all relations in the $\lambda$-frame, and we will omit the label ' $(\lambda)^{\prime}$. In the $\lambda$-frame, the derivative $\mathcal{D}_{\alpha}^{+}$is short, $\mathcal{D}_{\alpha}^{+}=D_{\alpha}^{+}$, and hence $V_{\alpha}^{+}=0$. However, two of the three harmonic derivatives acquire gauge connections:

$$
\mathcal{D}^{++}=D^{++}+\mathrm{i} V^{++}, \quad \mathcal{D}^{--}=D^{--}+\mathrm{i} V^{--} .
$$

As follows from the commutation relation $\left[\mathcal{D}_{\alpha}^{+}, \mathcal{D}^{++}\right]=0$, the gauge connection $V^{++}$ is analytic,

$$
D_{\alpha}^{+} V^{++}=0 .
$$

The connection $V^{--}$can be expressed via $V^{++}$as a unique solution of the zero-curvature condition

$$
\left[\mathcal{D}^{++}, \mathcal{D}^{--}\right]=D^{0} \quad \Longleftrightarrow \quad D^{++} V^{--}-D^{--} V^{++}+\mathrm{i}\left[V^{++}, V^{--}\right]=0
$$


The explicit expression for $V^{--}$in terms of $V^{++}$was originally found by Zupnik [44]. In the $\lambda$-frame, no $\tau$-gauge freedom remains. Under the $\lambda$-gauge group, the connections $V^{++}$ and $V^{--}$transform as

$$
V^{\prime \pm \pm}=\mathrm{e}^{\mathrm{i} \lambda} V^{ \pm \pm} \mathrm{e}^{-\mathrm{i} \lambda}-\mathrm{i} \mathrm{e}^{\mathrm{i} \lambda} D^{ \pm \pm} \mathrm{e}^{-\mathrm{i} \lambda}
$$

The $\lambda$-frame counterparts of the (anti-)commutation relations (A.3b) and (A.3c), in conjunction with the identity $\left[\mathcal{D}^{--}, \mathcal{D}_{\alpha}^{+}\right]=\mathcal{D}_{\alpha}^{-}$, allow one to express the gauge connections $V_{\alpha}^{-}$and $V_{a}$ and the field strength $W^{+\alpha}$ in terms of $V^{--}$. The explicit expressions for the connections are

$$
\begin{aligned}
V_{\alpha}^{-} & =-D_{\alpha}^{+} V^{--}, \\
V_{a} & =\frac{\mathrm{i}}{8}\left(\tilde{\gamma}_{a}\right)^{\alpha \beta} D_{\alpha}^{+} D_{\beta}^{+} V^{--} \quad \Longleftrightarrow \quad V_{\alpha \beta}=\left(\gamma^{a}\right)_{\alpha \beta} V_{a}=\frac{\mathrm{i}}{2} D_{\alpha}^{+} D_{\beta}^{+} V^{--} .
\end{aligned}
$$

The expression for the field strength is

$$
W^{+\alpha}=\frac{\mathrm{i}}{24} \varepsilon^{\alpha \beta \gamma \delta} D_{\beta}^{+} D_{\gamma}^{+} D_{\delta}^{+} V^{--} .
$$

As mentioned above, $V^{--}$is uniquely expressed in terms of the analytic connection $V^{++}$. Thus, the superfield $V^{++}$is a single prepotential in terms of which all the connections are determined, in complete analogy with the $4 \mathrm{D}$ case [44, 45]. This prepotential is analytic, but otherwise unconstrained.

Open Access. This article is distributed under the terms of the Creative Commons Attribution License (CC-BY 4.0), which permits any use, distribution and reproduction in any medium, provided the original author(s) and source are credited.

\section{References}

[1] J. Wess and B. Zumino, Consequences of anomalous Ward identities, Phys. Lett. 37B (1971) 95 [inSPIRE].

[2] K. Fujikawa, Path integral measure for gauge invariant fermion theories, Phys. Rev. Lett. 42 (1979) 1195 [INSPIRE].

[3] K. Fujikawa, Path integral for gauge theories with fermions, Phys. Rev. D 21 (1980) 2848 [Erratum ibid. D 22 (1980) 1499] [INSPIRE].

[4] R. Stora, Algebraic structure and topological origin of anomalies, in Recent progress in gauge theories, G. 't Hooft et al. eds., Plenum Press, New York U.S.A. (1984).

[5] B. Zumino, Chiral anomalies and differential geometry, in Relativity, groups and topology II, B.S. De Witt and R. Stora eds., North Holland, Amsterdam The Netherlands (1984).

[6] L. Álvarez-Gaumé and E. Witten, Gravitational anomalies, Nucl. Phys. B 234 (1984) 269 [INSPIRE].

[7] B. Zumino, Y.-S. Wu and A. Zee, Chiral anomalies, higher dimensions and differential geometry, Nucl. Phys. B 239 (1984) 477 [INSPIRE]. 
[8] W.A. Bardeen and B. Zumino, Consistent and covariant anomalies in gauge and gravitational theories, Nucl. Phys. B 244 (1984) 421 [INSPIRE].

[9] H. Leutwyler, Chiral fermion determinants and their anomalies, Phys. Lett. 152B (1985) 78 [INSPIRE].

[10] K. Fujikawa and H. Suzuki, Path integrals and quantum anomalies, Oxford University Press, Oxford U.K. (2004).

[11] F. Bastianelli and P. van Nieuwenhuizen, Path integrals and anomalies in curved space, Cambridge University Press, Cambridge U.S.A. (2006).

[12] P.K. Townsend and G. Sierra, Chiral anomalies and constraints on the gauge group in higher dimensional supersymmetric Yang-Mills theories, Nucl. Phys. B 222 (1983) 493 [INSPIRE].

[13] P.H. Frampton and T.W. Kephart, Explicit evaluation of anomalies in higher dimensions, Phys. Rev. Lett. 50 (1983) 1343 [Erratum ibid. 51 (1983) 232] [INSPIRE].

[14] P.H. Frampton and T.W. Kephart, Consistency conditions for Kaluza-Klein axial anomalies, Phys. Rev. Lett. 50 (1983) 1347 [INSPIRE].

[15] P.H. Frampton and T.W. Kephart, The analysis of anomalies in higher space-time dimensions, Phys. Rev. D 28 (1983) 1010 [InSPIRE].

[16] O. Piguet and K. Sibold, The anomaly in the Slavnov identity for $N=1$ supersymmetric Yang-Mills theories, Nucl. Phys. B 247 (1984) 484 [InSPIRE].

[17] T.E. Clark and S.T. Love, Supersymmetric effective actions for anomalous internal chiral symmetries, Phys. Lett. B 138 (1984) 289.

[18] N.K. Nielsen, Anomalies of supersymmetric chiral Yang-Mills currents, Nucl. Phys. B 244 (1984) 499 [INSPIRE].

[19] G. Girardi, R. Grimm and R. Stora, Chiral anomalies in $N=1$ supersymmetric Yang-Mills theories, Phys. Lett. B 156 (1985) 203.

[20] E. Guadagnini, K. Konishi and M. Mintchev, Non-abelian chiral anomalies in supersymmetric gauge theories, Phys. Lett. B 157 (1985) 37.

[21] K. Konishi and K. Shizuya, Functional integral approach to chiral anomalies in supersymmetric gauge theories, Nuovo Cim. A 90 (1985) 111 [INSPIRE].

[22] L. Bonora, P. Pasti and M. Tonin, ABJ anomalies in supersymmetric Yang-Mills theories, Phys. Lett. B 156 (1985) 341.

[23] L. Bonora, P. Pasti and M. Tonin, The consistent chiral anomaly in supersymmetric Yang-Mills theories, Nucl. Phys. B 261 (1985) 249 [Erratum ibid. B 269 (1986) 745] [INSPIRE].

[24] I.N. McArthur and H. Osborn, Supersymmetric chiral effective action and nonabelian anomalies, Nucl. Phys. B 268 (1986) 573 [INSPIRE].

[25] Y. Ohshima, K. Okuyama, H. Suzuki and H. Yasuta, Remark on the consistent gauge anomaly in supersymmetric theories, Phys. Lett. B 457 (1999) 291 [hep-th/9904096] [INSPIRE].

[26] M. Marinkovic, Wess-Zumino effective action for supersymmetric Yang-Mills theories, Nucl. Phys. B 366 (1991) 74 [INSPIRE]. 
[27] S.J. Gates, Jr., M.T. Grisaru, M.E. Knutt, S. Penati and H. Suzuki, Supersymmetric gauge anomaly with general homotopic paths, Nucl. Phys. B 596 (2001) 315 [hep-th/0009192] [INSPIRE].

[28] S. Ferrara, J. Wess and B. Zumino, Supergauge multiplets and superfields, Phys. Lett. 51B (1974) 239 [INSPIRE].

[29] P. Breitenlohner and M.F. Sohnius, Superfields, auxiliary fields and tensor calculus for $N=2$ extended supergravity, Nucl. Phys. B 165 (1980) 483 [INSPIRE].

[30] S.M. Kuzenko, J. Novak and I.B. Samsonov, The anomalous current multiplet in $6 D$ minimal supersymmetry, JHEP 02 (2016) 132 [arXiv: 1511.06582] [INSPIRE].

[31] P.S. Howe and E. Sezgin, Anomaly free tensor Yang-Mills system and its dual formulation, Phys. Lett. B 440 (1998) 50 [hep-th/9806050] [INSPIRE].

[32] P.S. Howe, G. Sierra and P.K. Townsend, Supersymmetry in Six-Dimensions, Nucl. Phys. B 221 (1983) 331 [INSPIRE].

[33] J. Koller, A six-dimensional superspace approach to extended superfields, Nucl. Phys. B 222 (1983) 319 [INSPIRE].

[34] W. Siegel, Superfields in higher dimensional space-time, Phys. Lett. 80B (1979) 220 [INSPIRE].

[35] B.E.W. Nilsson, Superspace action for a six-dimensional nonextended supersymmetric Yang-Mills theory, Nucl. Phys. B 174 (1980) 335 [INSPIRE].

[36] I.L. Buchbinder and N.G. Pletnev, Construction of $6 D$ supersymmetric field models in $N=(1,0)$ harmonic superspace, Nucl. Phys. B 892 (2015) 21 [arXiv:1411.1848] [InSPIRE].

[37] I.L. Buchbinder and N.G. Pletnev, Leading low-energy effective action in the $6 \mathrm{D}$ hypermultiplet theory on a vector/tensor background, Phys. Lett. B 744 (2015) 125 [arXiv: 1502.03257] [INSPIRE].

[38] I.L. Buchbinder, B.S. Merzlikin and N.G. Pletnev, Induced low-energy effective action in the $6 D, \mathcal{N}=(1,0)$ hypermultiplet theory on the vector multiplet background, Phys. Lett. B 759 (2016) 626 [arXiv : 1604.06186] [INSPIRE].

[39] I.L. Buchbinder, E.A. Ivanov, B.S. Merzlikin and K.V. Stepanyantz, One-loop divergences in the $6 D, \mathcal{N}=(1,0)$ abelian gauge theory, Phys. Lett. B 763 (2016) 375 [arXiv:1609.00975] [INSPIRE].

[40] I.L. Buchbinder, E.A. Ivanov, B.S. Merzlikin and K.V. Stepanyantz, One-loop divergences in $6 D, \mathcal{N}=(1,0)$ SYM theory, JHEP 01 (2017) 128 [arXiv:1612.03190] [InSPIRE].

[41] I.L. Buchbinder, E.A. Ivanov, B.S. Merzlikin and K.V. Stepanyantz, Supergraph analysis of the one-loop divergences in $6 D, \mathcal{N}=(1,0)$ and $\mathcal{N}=(1,1)$ gauge theories, Nucl. Phys. B 921 (2017) 127 [arXiv: 1704.02530] [INSPIRE].

[42] P.S. Howe, K.S. Stelle and P.C. West, $N=1 D=6$ harmonic superspace, Class. Quant. Grav. 2 (1985) 815 [INSPIRE].

[43] B.M. Zupnik, Six-dimensional supergauge theories in the harmonic superspace, Sov. J. Nucl. Phys. 44 (1986) 512 [Yad. Fiz. 44 (1986) 794] [INSPIRE].

[44] B.M. Zupnik, The action of the supersymmetric $N=2$ gauge theory in harmonic superspace, Phys. Lett. B 183 (1987) 175 [INSPIRE]. 
[45] A. Galperin, E. Ivanov, S. Kalitsyn, V. Ogievetsky and E. Sokatchev, Unconstrained $N=2$ Matter, Yang-Mills and supergravity theories in harmonic superspace, Class. Quant. Grav. 1 (1984) 469 [Erratum ibid. 2 (1985) 127] [INSPIRE].

[46] A.S. Galperin, E.A. Ivanov, V.I. Ogievetsky and E.S. Sokatchev, Harmonic superspace, Cambridge University Press, Cambrdige U.K. (2001).

[47] L. Mezincescu, On the superfield formulation of O(2) supersymmetry, Dubna preprint JINR-P2-12572 (1979).

[48] I.L. Buchbinder, E.I. Buchbinder, S.M. Kuzenko and B.A. Ovrut, The background field method for $N=2$ super Yang-Mills theories in harmonic superspace, Phys. Lett. B 417 (1998) 61 [hep-th/9704214] [INSPIRE].

[49] I.L. Buchbinder and S.M. Kuzenko, Comments on the background field method in harmonic superspace: nonholomorphic corrections in $N=4$ SYM, Mod. Phys. Lett. A 13 (1998) 1623 [hep-th/9804168] [INSPIRE].

[50] S.M. Kuzenko and I.N. McArthur, Effective action of $N=4$ super Yang-Mills: $N=2$ superspace approach, Phys. Lett. B 506 (2001) 140 [hep-th/0101127] [INSPIRE].

[51] S.M. Kuzenko and I.N. McArthur, Hypermultiplet effective action: $N=2$ superspace approach, Phys. Lett. B 513 (2001) 213 [hep-th/0105121] [INSPIRE].

[52] S.M. Kuzenko, Five-dimensional supersymmetric Chern-Simons action as a hypermultiplet quantum correction, Phys. Lett. B 644 (2007) 88 [hep-th/0609078] [INSPIRE].

[53] W.D. Linch, III and G. Tartaglino-Mazzucchelli, Six-dimensional supergravity and projective superfields, JHEP 08 (2012) 075 [arXiv: 1204.4195] [INSPIRE].

[54] L. Bonora, P. Pasti and M. Tonin, Chiral anomalies in higher dimensional supersymmetric theories, Nucl. Phys. B 286 (1987) 150 [InSPIRE].

[55] B. de Wit and M.T. Grisaru, Compensating fields and anomalies, in Quantum Field Theory and Quantum Statistics, Volume 2, I.A. Batalin et al. eds.,, Adam Hilger, Bristol, U.K. (1987).

[56] S.M. Kuzenko and W.D. Linch III, On five-dimensional superspaces, JHEP 02 (2006) 038 [hep-th/0507176] [INSPIRE].

[57] A. Galperin, E.A. Ivanov, V. Ogievetsky and E. Sokatchev, Harmonic supergraphs. Green functions, Class. Quant. Grav. 2 (1985) 601 [INSPIRE].

[58] E.A. Ivanov, A.V. Smilga and B.M. Zupnik, Renormalizable supersymmetric gauge theory in six dimensions, Nucl. Phys. B 726 (2005) 131 [hep-th/0505082] [INSPIRE].

[59] A.V. Smilga, Chiral anomalies in higher-derivative supersymmetric $6 D$ theories, Phys. Lett. B 647 (2007) 298 [hep-th/0606139] [INSPIRE].

[60] A. Davgadorj and R. von Unge, $\mathcal{N}=2$ super Yang-Mills theory in projective superspace, arXiv: 1706.07000 [INSPIRE]. 